\title{
Ubiquitous $\mathrm{NH}_{3}$ supersonic component in L1688 coherent cores
}

\author{
Spandan Choudhury ${ }^{1}$, Jaime E. Pineda ${ }^{1}$, Paola Caselli ${ }^{1}$, Adam Ginsburg ${ }^{2}$, Stella S. R. Offner ${ }^{3}$, Erik Rosolowsky ${ }^{4}$, \\ Rachel K. Friesen ${ }^{5}$, Felipe O. Alves ${ }^{1}$, Ana Chacón-Tanarro ${ }^{6}$, Anna Punanova ${ }^{7}$, Elena Redaelli ${ }^{1}$, Helen Kirk ${ }^{8,9}$, \\ Philip C. Myers ${ }^{10}$, Peter G. Martin ${ }^{11}$, Yancy Shirley ${ }^{12}$, Michael Chun-Yuan Chen ${ }^{8}$,
} Alyssa A. Goodman ${ }^{10}$, and James Di Francesco ${ }^{13}$

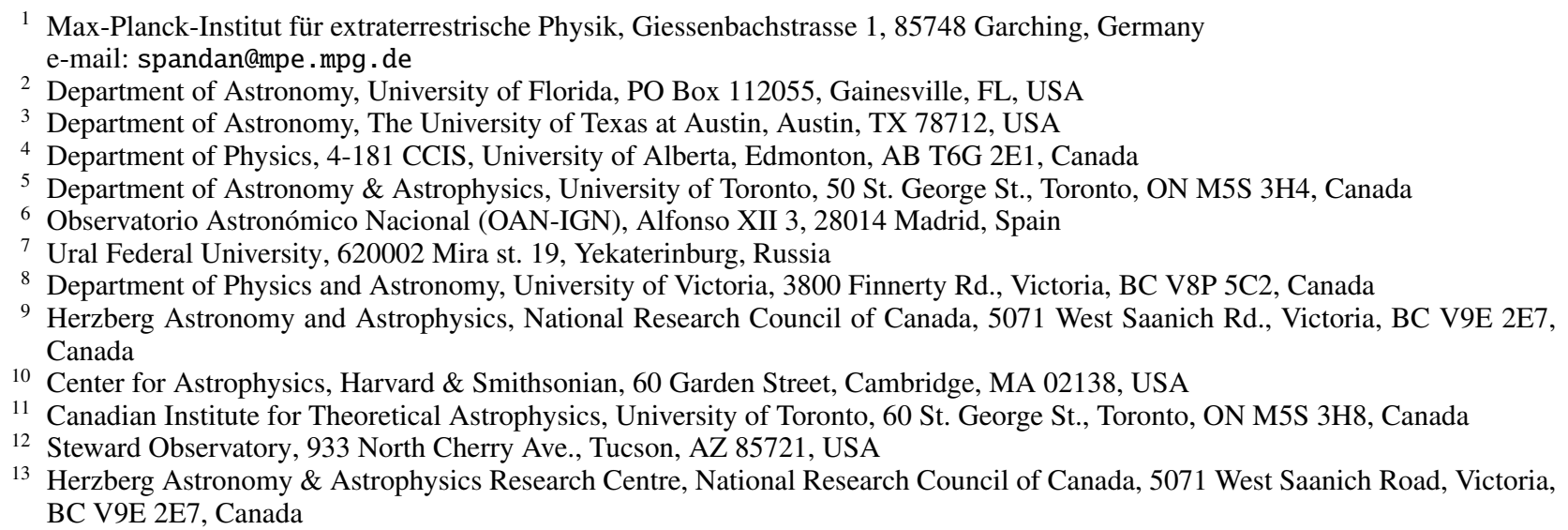

Received 13 March 2020 / Accepted 6 July 2020

\begin{abstract}
Context. Star formation takes place in cold dense cores in molecular clouds. Earlier observations have found that dense cores exhibit subsonic non-thermal velocity dispersions. In contrast, $\mathrm{CO}$ observations show that the ambient large-scale cloud is warmer and has supersonic velocity dispersions.

Aims. We aim to study the ammonia $\left(\mathrm{NH}_{3}\right)$ molecular line profiles with exquisite sensitivity towards the coherent cores in L1688 in order to study their kinematical properties in unprecedented detail.

Methods. We used $\mathrm{NH}_{3}(1,1)$ and $(2,2)$ data from the first data release (DR1) in the Green Bank Ammonia Survey (GAS). We first smoothed the data to a larger beam of $1^{\prime}$ to obtain substantially more extended maps of velocity dispersion and kinetic temperature, compared to the DR1 maps. We then identified the coherent cores in the cloud and analysed the averaged line profiles towards the cores.

Results. For the first time, we detected a faint (mean $\mathrm{NH}_{3}(1,1)$ peak brightness $<0.25 \mathrm{~K}$ in $T_{\mathrm{MB}}$ ), supersonic component towards all the coherent cores in L1688. We fitted two components, one broad and one narrow, and derived the kinetic temperature and velocity dispersion of each component. The broad components towards all cores have supersonic linewidths $\left(\mathcal{M}_{S} \geq 1\right)$. This component biases the estimate of the narrow dense core component's velocity dispersion by $\approx 28 \%$ and the kinetic temperature by $\approx 10 \%$, on average, as compared to the results from single-component fits.

Conclusions. Neglecting this ubiquitous presence of a broad component towards all coherent cores causes the typical singlecomponent fit to overestimate the temperature and velocity dispersion. This affects the derived detailed physical structure and stability of the cores estimated from $\mathrm{NH}_{3}$ observations.
\end{abstract}

Key words. ISM: kinematics and dynamics - ISM: molecules - stars: formation - ISM: individual objects: L1688, Ophiuchus

\section{Introduction}

Star formation takes place in dense cores, which are embedded in molecular clouds. These cores in various molecular clouds are therefore studied in detail in relation to their physical and chemical properties. Across different molecular clouds, the starforming cores are characterised by higher density and lower temperatures, as compared to the parental cloud (Myers 1983; Myers \& Benson 1983; Caselli et al. 2002). When studied using molecular transitions tracing higher densities $\left(n\left(\mathrm{H}_{2}\right)>10^{4} \mathrm{~cm}^{-3}\right)$, the cores are revealed to show subsonic levels of turbulence (Barranco \& Goodman 1998; Kirk et al. 2007; Rosolowsky et al. 2008). This is in contrast to lower density gas surrounding the cores, which show supersonic linewidths (Goodman et al. 1998).

Thanks to the numerous hyperfine components, $\mathrm{NH}_{3}$ remains optically thin in the individual components even at high column densities (see Caselli et al. 2017), thereby making it an important and useful tracer of cold and dense gas. Barranco \& Goodman (1998) studied four cores in $\mathrm{NH}_{3}(1,1)$ emission, and found that the linewidths within the cores were roughly constant 
and slightly greater than the pure thermal value. They called these "coherent cores". A "transition to coherence" from the turbulent environment to the coherent region was hypothesised (Goodman et al. 1998), but not directly observed. Williams \& Myers (2000) also noted eight "quiescent" cores, which they defined as local minima in non-thermal dispersion, in the observation of Serpens in $\mathrm{N}_{2} \mathrm{H}^{+}$, using BIMA (Berkeley Illinois Maryland Association). They showed that the dispersion increased outwards from the centres of these cores. Using $\mathrm{NH}_{3}(1,1)$ observations with the Green Bank Telescope (GBT), Pineda et al. (2010) studied the transition from the surrounding gas to inside a core, for the first time in the same tracer in the B5 region in Perseus, and reported a sharp transition to coherence (a decrease in the velocity dispersion by a factor of 2 within $0.04 \mathrm{pc}$ ). This suggests that we can define the boundaries of coherent cores in a systematic fashion, as regions with subsonic non-thermal linewidths (see also Chen et al. 2019). It should be noted that this definition of cores does not necessarily define cores identically to those defined using continuum emission. Therefore, not all of the "coherent cores" have continuum counterparts.

In the Green Bank Ammonia Survey (GAS; Friesen et al. 2017), star forming regions in the Gould Belt were observed using $\mathrm{NH}_{3}$ hyperfine transitions. The first data release (DR1) includs the following four regions in nearby molecular clouds: B18 in Taurus, NGC 1333 in Perseus, L1688 in Ophiuchus, and Orion A North. Using the results from this survey and $\mathrm{H}_{2}$ column densities derived from Herschel data, Chen et al. (2019) identified 12 coherent structures (termed "droplet" in the paper) in L1688 and six in B18. They observed that these droplets show high gas density $\left(\left\langle n_{\mathrm{H}_{2}}\right\rangle \approx 5 \times 10^{4} \mathrm{~cm}^{-3}\right.$, derived from their masses and radii) and near-constant, almost-thermal velocity dispersions, with a sharp velocity dispersion increase across the boundary.

This Letter presents a new analysis of the GAS DR1 observations to reach unprecedented noise levels towards coherent cores and reveal a broad supersonic component that has been previously unidentified. We focus on L1688, which is part of the Ophiuchus molecular cloud (distance $=138.4 \pm 2.6 \mathrm{pc}$, OrtizLeón et al. 2018, and mass $\sim 980 M_{\odot}$, Ladjelate et al. 2020) because it is one of the nearest star-forming regions and with the most extended $\mathrm{NH}_{3}$ emission beyond cores among the GAS DR1 regions.

\section{Ammonia maps}

We use the $\mathrm{NH}_{3}(1,1)$ and $(2,2)$ maps from GAS DR1 (Friesen et al. 2017). The observations were carried out using the GBT to map $\mathrm{NH}_{3}$ emission in the star-forming regions in the Gould Belt with $A_{V}>7$ mag using the seven-beam $K$-band Focal Plane Array (KFPA) at the GBT. Observations were performed in frequency switching mode with a frequency throw of 4.11 $\mathrm{MHz}\left(\approx 52 \mathrm{~km} \mathrm{~s}^{-1}\right.$ at $\left.23.7 \mathrm{GHz}\right)$. The spectral resolution of the data is $5.7 \mathrm{kHz}$, which corresponds to $\approx 72.1 \mathrm{~m} \mathrm{~s}^{-1}$ at $23.7 \mathrm{GHz}$ (i.e. the approximate frequency of observations). The extents of the maps were selected using continuum data from Herschel or JCMT, or extinction maps derived from 2MASS (Two Micron All Sky Survey). To convert the spectra from frequency to velocity space, the rest frequencies for $\mathrm{NH}_{3}(1,1)$ and $(2,2)$ lines were considered to be $23.6944955 \mathrm{GHz}$ and $23.7226336 \mathrm{GHz}$, respectively.

The parameter maps of L1688, which were released in DR1, are very patchy, particularly for the kinetic temperature. Therefore, to obtain a good estimate of the Mach number throughout the cloud, which requires determining the temperature, and thus, a good detection of the $(2,2)$ line, the data were smoothed by convolving them to a beam of $1^{\prime}$ (GBT native beam at $23 \mathrm{GHz}$ is $\left.\approx 31^{\prime \prime}\right)$. The data cube was then re-gridded to avoid oversampling. The relative pixel size was kept the same as in the original GAS maps, at one-third the beam width. The median noise level achieved as a result is $39 \mathrm{mK}$ in the regions of interest (see Sects. 3.2 and 3.3). In comparison, the median noise in the same regions was $131 \mathrm{mK}$ in the original GAS DR 1 maps ${ }^{1}$.

\section{Analysis}

\subsection{Line fitting}

We fitted $\mathrm{NH}_{3}$ line profiles to the data with the pyspeckit package (Ginsburg \& Mirocha 2011), which uses a forward modelling approach, following the process described in Friesen et al. (2017). The range in velocity to fit is determined from the average spectrum of the entire region (Fig. F.1). Since the ortho-topara ratio in the region is not known, we only used the $p-\mathrm{NH}_{3}$ column density in the fitting process, and did not attempt to convert it to total $\mathrm{NH}_{3}$ column density ${ }^{2}$.

The model produces synthetic spectra based on initial guesses provided for the input parameters: excitation temperature $\left(T_{\mathrm{ex}}\right)$, kinetic temperature $\left(T_{\mathrm{K}}\right)$, para-ammonia column density $\left(N\left(p-\mathrm{NH}_{3}\right)\right)$, velocity dispersion $\left(\sigma_{\mathrm{v}}\right)$, and line-of-sight central velocity $\left(v_{\mathrm{LSR}}\right)$ of the gas (Sect. 3.1 in Friesen et al. 2017). A non-linear gradient descent algorithm, MPFIT (Markwardt 2009) is then used to determine the best-fit model, and the corresponding values of the parameters. A good set of initial conditions is necessary to ensure that the non-linear least-squares fitting does not get stuck in a local minimum. The value of $v_{\mathrm{LSR}}$ is critical, and, therefore, we used the first-order moments of the $(1,1)$ line as initial guesses. The second-order moments were used as guesses for velocity dispersion. For the other parameters, we used guesses based on the GAS DR1 map of the respective parameter. The initial guesses used in our work are: $\log _{10}\left(N_{p-\mathrm{NH}_{3}} / \mathrm{cm}^{-2}\right)=14, T_{\mathrm{K}}=20 \mathrm{~K}$, and $T_{\mathrm{ex}}=5 \mathrm{~K}$. These numbers are within the range of the values reported in the GAS DR1 maps, and therefore, they are reasonable guesses. As a test, we checked the fits with varying guesses and determined that the exact values of the initial parameters did not affect the final results, as long as they were within a reasonable range.

In this work, we use the cold_ammonia model in the pyspeckit library, which makes the assumption that only the $(1,1),(2,2)$ and $(2,1)$ levels are occupied. It is also assumed that the excitation temperature, $T_{\mathrm{ex}}$, is the same for the $(1,1)$ and $(2,2)$ transitions, as well as their hyperfine components.

\subsection{Identification of coherent cores}

To study the spectra towards cores, we first define "coherent cores" as the region with a sonic Mach number $<1$ and larger

1 The GAS data use a tapered Bessel function as the gridding kernel (advocated for by Mangum et al. 2007), to achieve maximum resolution. The kernel is not strictly positive, which leads to adjacent pixels having anti-correlated noise. When smoothing over anti-correlated values, the noise level drops faster than what is expected from independent data. Therefore, we see the noise level drop faster than the inverse of the beam radius.

2 To compare $N(p-\mathrm{NH} 3)$ to the total $\mathrm{NH}_{3}$ column densities reported in other works, an easy conversion is to multiply it by two, with the ortho-to-para ratio assumed to be unity. 


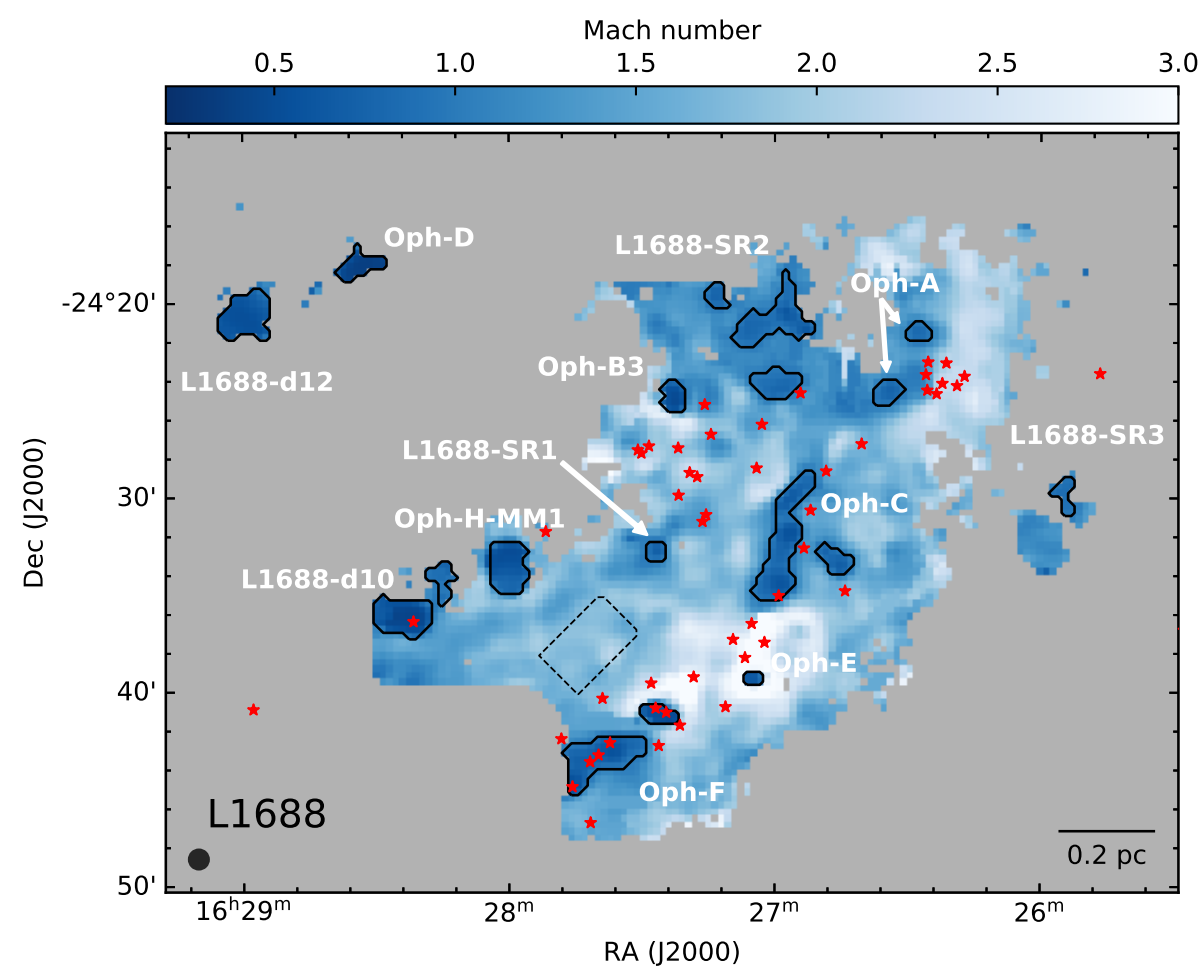

Fig. 1. Coherent cores, as defined in Sect. 3.2, are shown by black contours. The background colour scale shows the sonic Mach number. The dashed rectangle shows the box considered to be representative of the ambient cloud. The red stars show positions of Class 0/I and flat-spectrum protostars in the cloud (Dunham et al. 2015). The beam and the scale bar are shown in the bottom left and bottom right corners, respectively. than a beam (similar to Pineda et al. 2010; Chen et al. 2019) ${ }^{3}$. The sonic Mach number, $\mathcal{M}_{\mathcal{S}}$, is defined as the ratio of the nonthermal velocity dispersion, $\sigma_{\mathrm{NT}}$ (see Appendix A) to the sound speed in the medium,

$\mathcal{M}_{\mathcal{S}}=\frac{\sigma_{\mathrm{NT}}}{c_{S}}$

where $c_{S}$ is the one-dimensional sound speed in the gas,

$c_{S}=\sqrt{\frac{k_{\mathrm{B}} T_{\mathrm{K}}}{\mu_{\mathrm{gas}}}}$.

Here $k_{\mathrm{B}}$ is the Boltzmann's constant, $T_{\mathrm{K}}$ is the kinetic temperature in the region, and $\mu_{\text {gas }}=2.37 \mathrm{amu}$ is the average molecular mass (Kauffmann et al. 2008).

Based on this definition, we identify 12 regions in L1688 as coherent cores. They are shown in Fig. 1. See Appendix B for a brief description of the cores.

\subsection{Averaging spectra towards the coherent cores}

We first averaged the spectra towards each coherent core defined in Sect. 3.2, to get a higher signal-to-noise ratio. To avoid any possible line broadening due to averaging in a region with velocity gradients, we aligned the spectra at each pixel within a core before averaging. We took the velocity at a pixel, which was determined from the single-component fit at that pixel, and using the channelShift function from module gridregion in the GAS pipeline ${ }^{4}$, we shifted the spectra by the corresponding num-

\footnotetext{
3 It should be noted that Chen et al. (2019) also require a corresponding peak in $\mathrm{N}\left(\mathrm{H}_{2}\right)$ inside a coherent region for it to be identified as a "droplet", whereas we consider the sonic Mach number as the only criterion.

4 https://github.com/GBTAmmoniaSurvey/GAS/tree/master/ GAS
}

ber of channels. Then, we averaged the resultant spectra from all pixels inside a core, which are now essentially aligned at $v=0$. This averaging allowed us to reach a typical noise level of $18 \mathrm{mK}$ in the average spectra, which is almost seven times better than the median noise level of $131 \mathrm{mK}$ in the individual spectra towards the cores in the original GAS maps.

We checked if smoothing the data (Sect. 2) before averaging had any effect on the results and found that the change is not significant. See Appendix C for details.

\section{Results}

\subsection{Presence of a second component in core spectra}

The top panels in Fig. 2 show the average spectra in the OphF core, with a single-component fit (see Appendix D for the guesses used in the fit process), and corresponding residual. Oph-F is shown here as an example, the average spectra for the other coherent cores are shown in Figs. G.1-G.11. From the residuals, it is evident that the fit does not recover all of the flux at the positions of the hyperfine lines. In particular, the singlecomponent fit misses the flux present in a broad component, which is ubiquitously present towards all cores.

Therefore, we added a second component to the fit (see Appendix D for the fit procedure). The resultant two-component fits and the individual components for Oph-F are shown in the bottom panel of Fig. 2, and in Figs. G.1-G.11 for the other cores. The two-component fit is clearly an improvement (better $\chi^{2}$ ), as seen from the respective residuals. We employed the Akaike Information Criterion (AIC, see Appendix E) to verify that for each core, considering a second component corresponds to a significant improvement in the fit to the spectra. The difference in AIC values from single-component fits to two-component fits, $\Delta_{\mathrm{AIC}}=\mathrm{AIC}_{1-\text { comp. }}-\mathrm{AIC}_{2 \text {-comp. }}$, are shown in Table H.1.

In model comparison, the model with the lowest AIC value is the preferred one, and, therefore all regions are better fit by a 

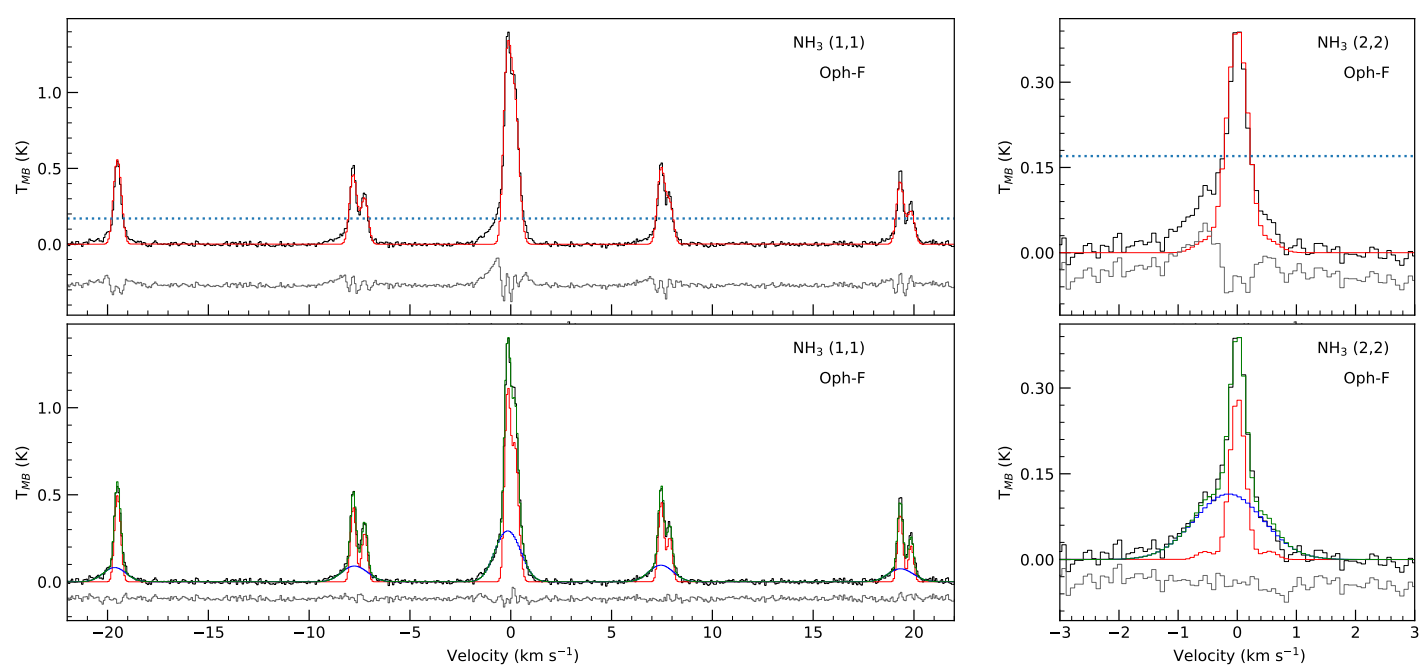

Fig. 2. Top panels: average $\mathrm{NH}_{3}(1,1)$ and $(2,2)$ spectra of Oph-F core, with a single-component fit. The blue-dotted line shows the median noise level in Oph-F in the original GAS data, which clearly shows that the broad component cannot be detected in the individual spectra in GAS DR1 data. Bottom panels: same spectra, with two-component fit (green). The narrow (red) and broad (blue) components are also shown separately.

two-component fit. However, for coherent cores L1688-d12 and L1688-SR3, the value of $\Delta_{\mathrm{AIC}}$ is small (see Table H.1) and the average spectra fits (Figs. G.8 and G.11) only show marginal improvements with the two-component fit. Therefore, we note that these two regions are better fit with a two-component model, but their fit-derived results are not as well-constrained as in the other ten coherent cores.

The kinetic temperatures, velocity dispersions, LSR velocities, and $p-\mathrm{NH}_{3}$ column densities for the single-component fits and for each individual component in the two-component fits are shown in Table H.1. We see that one of the two components is subsonic $\left(\mathcal{M}_{S}<1\right)$ across all cores, whereas the other component has $\mathcal{M}_{S} \geq 1$. We refer to the subsonic and supersonic components as "narrow" and "broad" components, respectively.

We note that the residuals from the two-component fit in some cores, in particular Oph-E, might suggest the presence of a third component or a non-Gaussian component. However, we do not see this towards all of the cores. Even when this occurs, the indication is not very clear: residuals comparable to the noise or not more than one or two channels wide. Therefore, we did not fit more than two components to the spectra in the cores.

\subsection{Detection of a broad component in cores}

A narrow and a broad component of ammonia emission has been detected in all of the coherent cores in L1688 (Fig. 1). The broad component in the cores is fainter, with a mean $(1,1)$ peak brightness temperature $\left(T_{\mathrm{p}}\right) \approx 0.25 \mathrm{~K}$ and $T_{\mathrm{p},(2,2)}<0.13 \mathrm{~K}$, except in Oph-A $\left(T_{\mathrm{p},(2,2)} \approx 0.27 \mathrm{~K}\right)$ and Oph-C $\left(T_{\mathrm{p},(2,2)} \approx 0.16 \mathrm{~K}\right)$. Because of such low intensities, this component cannot be detected from the previous ammonia maps. As a comparison, the original GAS maps had noise levels of $\approx 131 \mathrm{mK}$ in the coherent core regions, which is not low enough for a $3 \sigma$ detection of the $(1,1)$, and in most cases, this is comparable to the $T_{\mathrm{MB}}$ of the broad component in the $(2,2)$. Therefore, using the original GAS data, one would not be able to detect the broad component, and the temperature and linewidth of the narrow core component would be affected. As can be seen in Table H.1, the mean noise level in the average spectra in the cores is $\approx 18 \mathrm{mK}$ in this work, enabling us to comfortably fit even the faint broad component in these spectra for both $(1,1)$ and $(2,2)$.

\subsection{Spectra in the ambient cloud outside cores}

To check if the two components, which are seen towards the coherent cores, are present throughout L1688, we looked at the spectra outside of the cores. Since the spectra outside of the cores are even fainter than those inside of them, we have to consider the average spectra in a small region, away from the cores. Therefore, we defined a rectangular box (shown by the blackdashed box in Fig. 1) that is representative of the ambient cloud in L1688, as :

- being at least two beams away from any coherent cores, to avoid contamination from the core spectra;

- not including, or being very $\operatorname{close}(\lesssim$ one beam) to known protostars; and

- being away from the western edge of the cloud, which is affected by a strong external illumination (Habart et al. 2003).

The top panels of Fig. 3 show the average spectrum in the ambient cloud with a single-component fit. Even when a twocomponent fit was attempted, we did not see a narrow component (bottom panel of Fig. 3). AIC indicates that the twocomponent fit is a better model, with both components being supersonic (Mach number $>1$ ). Therefore, we conclude that these are two broad components in the ambient cloud, which are separated in centroid velocity by $\approx 0.6 \mathrm{~km} \mathrm{~s}^{-1}$. The two components in the spectra have equal dispersions of $0.35 \mathrm{~km} \mathrm{~s}^{-1}$. The kinetic temperatures of these two components are also approximately equal, $16.9 \pm 0.2 \mathrm{~K}$ and $17.0 \pm 0.5 \mathrm{~K}$. These values are comparable to those from the broad components in the cores, and higher than the typical temperature of the narrow components. The Mach numbers of these two cloud components are comparable to that of the broad components seen in the cores (Table H.1).

The residuals from the single-component fit to the ambient cloud spectra are not as suggestive as in the cores. Therefore, the single-component model can also be considered as a reasonable fit. Even then, the comparison between the ambient cloud and the broad component towards the cores, as mentioned above, remains the same. The temperature, velocity dispersion, and the Mach number in the ambient cloud, from a single-component fit, are very similar to those in the broad component in the coherent cores. 

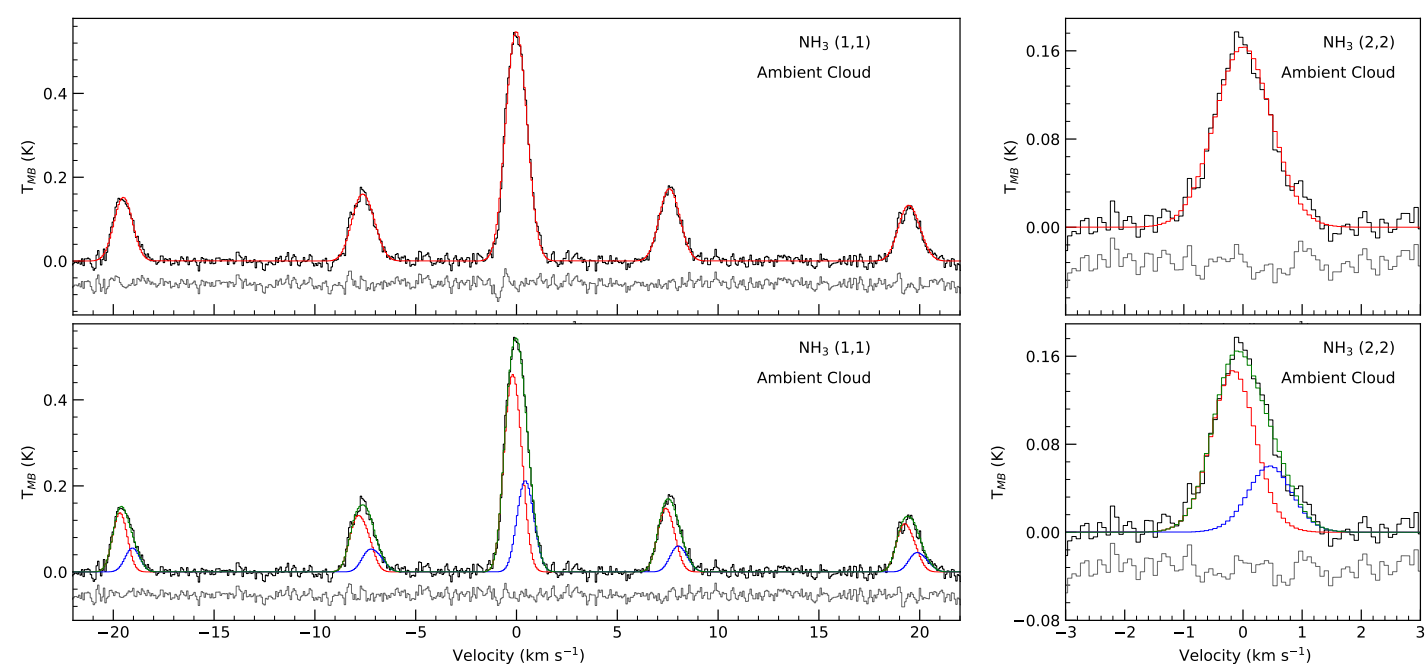

Fig. 3. Top panels: average spectra of ambient cloud (defined in Sect. 4.3) with a single-component fit. Bottom panels: same spectra, with twocomponent fit (green). The individual components are shown separately in red and blue.

\subsection{Comparison of physical parameters determined from the fit}

Figure 4 shows the comparison between kinetic temperature, velocity dispersion, Mach number, and non-thermal dispersion in the coherent cores, from a single-component fit, and for the narrow component in the two-component fit. Clearly, a singlecomponent fit significantly overestimates all of these parameters in all cores. The kinetic temperature from the narrow component is lower by $1.19 \pm 0.07 \mathrm{~K}$, compared to the single-component fit. Similarly, the velocity dispersion of the narrow component is lower by an average $\mathrm{e}^{5} 0.0332 \pm 0.0007 \mathrm{~km} \mathrm{~s}^{-1}$. The difference is as high as $\approx 0.1 \mathrm{~km} \mathrm{~s}^{-1}$ in the case of SR1. On average, the single-component fit overestimates the kinetic temperature by $10 \%$ and the velocity dispersion by $28 \%$, as compared to the narrow component. Correspondingly, the Mach number is overestimated, on average, by $32 \%$, and the non-thermal dispersion is typically overestimated by $39 \%$.

The average kinetic temperatures and velocity dispersions inside the cores in the GAS DR1 parameter maps, which were obtained using single-component fits, are very similar to the single-component results we report. Therefore, the aforementioned comparison with narrow component results still holds, if the DR1 results (final two columns in Table H.1) are considered. On average, the GAS DR1 results show a $14 \%$ overestimate in $T_{\mathrm{K}}$ and a $34 \%$ overestimate in $\sigma_{\mathrm{v}}$, as compared to our narrow component results.

\section{Discussion and conclusions}

For the first time, a faint, broad component has been detected towards coherent dense cores. When we consider a twocomponent fit to the spectra, it enables us to measure the velocity dispersion and kinetic temperature of the coherent core more accurately. We find that the typically-used single-component fit towards dense cores overestimates their temperatures and dispersions, on average, by $10 \%$ and $28 \%$, respectively.

The $1.2 \mathrm{~K}$ systematic offset in temperature in the cores derived from the single-component fit is important, as it is comparable to the temperature drop observed towards the centre of the cores when compared to the outer 1' or 2' (Crapsi et al. 2007;

\footnotetext{
5 Weighted averages of $\Delta T_{\mathrm{K}}$ and $\Delta \sigma_{\mathrm{v}}$.
}

Harju et al. 2017). This decreased temperature of the cores has an effect on the chemistry inside the cores (Caselli \& Ceccarelli 2012; Bergin \& Tafalla 2007), since, at the volume densities of L1688 dense cores $\left(\sim 10^{5} \mathrm{~cm}^{-3}\right)$, gas and dust are thermally coupled (Goldsmith 2001) and surface chemistry rates exponentially depend on the dust temperature (see Hasegawa et al. 1992).

Without the detection of the faint broad component, both the temperature and velocity dispersion in the cores are overestimated. This changes the estimates of the dynamical stability for the cores.

In the ambient cloud surrounding the coherent cores, we do not see the narrow component. Instead, at the position of our "ambient cloud box" (Fig. 1), we see two broad components that are $\approx 0.6 \mathrm{~km} \mathrm{~s}^{-1}$ apart in centroid velocity, with equal temperatures and velocity dispersions. This might suggest the presence of two molecular clouds at slightly different velocities, which are probably merging at the location of L1688. The collision between these two clouds might result in a local density increase, where the narrow features are produced following a corresponding dissipation of turbulence, thus creating the observed coherent cores with subsonic linewidths.

On the other hand, if the faint broad features seen towards the coherent core position trace the less dense and more turbulent cloud along the line of sight of the coherent core, then we can measure the relative motions between the cores and the cloud in a direct way. Accordingly, we measured the relative velocity between the narrow and broad component, $\delta v_{(n-b)}$. Twothirds of the coherent cores show subsonic $\left|\delta v_{(n-b)}\right|$ values, which suggests that most cores are dynamically coupled to their natal cloud (Kirk et al. 2010). The standard deviation of $\delta v_{(n-b)}$ is $0.35 \mathrm{~km} \mathrm{~s}^{-1}$, which is lower than the broad component's typical velocity dispersion $\left(0.48 \mathrm{~km} \mathrm{~s}^{-1}\right)$. Therefore, the motions of the cores are lower than the typical motions in the surrounding gas (see also Walsh et al. 2007; Kirk et al. 2007, 2010). Bailey et al. (2015) have also reported similar findings in simulated observations.

The cloud velocity dispersions that we derived from $\mathrm{NH}_{3}$ are lower than those obtained using lower density tracers (CO) in the earlier studies. Therefore, the results from lower density tracers might overestimate the local degree of turbulence in the cloud, or $\mathrm{NH}_{3}$ based measurements provide a strict lower bound to it. 

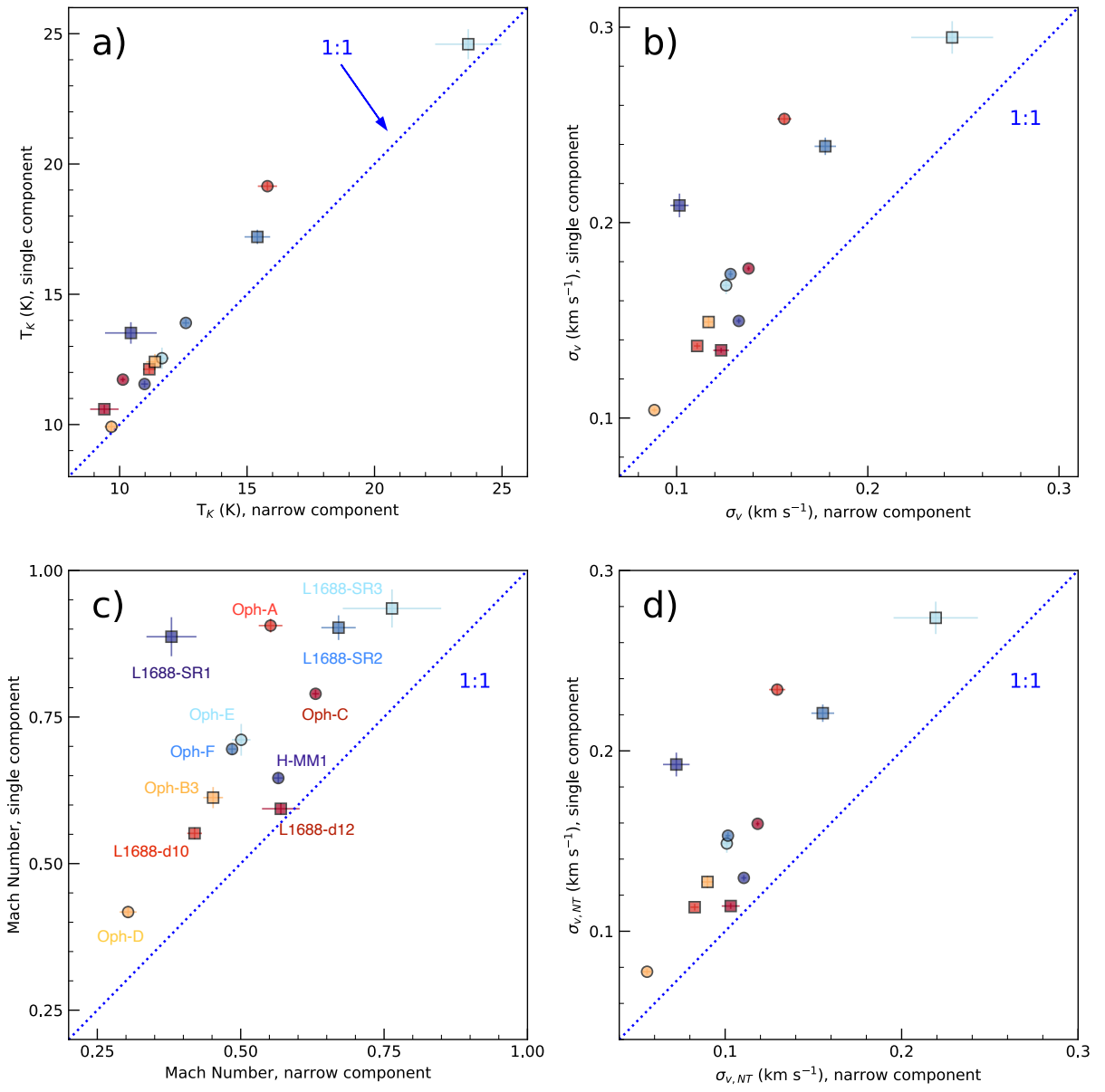

Fig. 4. Kinetic temperature, velocity dispersion, Mach number and non-thermal dispersion in the cores, from single-component fits, and for the narrow component of a twocomponent fit, are shown in panels $a-d$, respectively. In the cases where the error bar is not visible, the error is smaller than the symbols.

Without the required sensitivity in the observations, the faint broad component is not detected, and, therefore cannot be considered in the fit. This causes both the kinetic temperature and the velocity dispersion in the cores to be overestimated. Therefore, our results suggest that with deeper observations, we obtain better estimates of the core properties.

Acknowledgements. AP is supported by the Russian Ministry of Science and Higher Education via the State Assignment Project FEUZ-2020-0038. AP is a member of the Max Planck Partner Group at the Ural Federal University. SSRO acknowledges support from NSF CAREER grant. AC-T acknowledges support from MINECO project AYA2016-79006-P.

\section{References}

Bailey, N. D., Basu, S., \& Caselli, P. 2015, ApJ, 798, 75

Barranco, J. A., \& Goodman, A. A. 1998, ApJ, 504, 207

Bergin, E. A., \& Tafalla, M. 2007, ARA\&A, 45, 339

Caselli, P., \& Ceccarelli, C. 2012, A\&ARv, 20, 56

Caselli, P., Benson, P. J., Myers, P. C., \& Tafalla, M. 2002, ApJ, 572, 238

Caselli, P., Bizzocchi, L., Keto, E., et al. 2017, A\&A, 603, L1

Chen, H. H.-H., Pineda, J. E., Goodman, A. A., et al. 2019, ApJ, 877, 93

Crapsi, A., Caselli, P., Walmsley, M. C., \& Tafalla, M. 2007, A\&A, 470, 221

Di Francesco, J., André, P., \& Myers, P. C. 2004, ApJ, 617, 425

Dunham, M.M., Allen, L.E., Evans, I., \& Neal, J. 2015, ApJS, 220, 11

Friesen, R. K., Di Francesco, J., Shirley, Y. L., \& Myers, P. C. 2009, ApJ, 697, 1457
Friesen, R. K., Pineda, J. E., co-PIs, et al. 2017, ApJ, 843, 63

Ginsburg, A., \& Mirocha, J. 2011, PySpecKit: Python Spectroscopic Toolkit

Goldsmith, P. F. 2001, ApJ, 557, 736

Goodman, A. A., Barranco, J. A., Wilner, D. J., \& Heyer, M. H. 1998, ApJ, 504, 223

Habart, E., Boulanger, F., Verstraete, L., et al. 2003, A\&A, 397, 623

Harju, J., Daniel, F., Sipilä, O., et al. 2017, A\&A, 600, A61

Hasegawa, T. I., Herbst, E., \& Leung, C. M. 1992, ApJS, 82, 167

Johnstone, D., Di Francesco, J., \& Kirk, H. 2004, ApJ, 611, L45

Kauffmann, J., Bertoldi, F., Bourke, T. L., Evans, N. J. I., \& Lee, C. W. 2008, A\&A, 487, 993

Kirk, H., Johnstone, D., \& Tafalla, M. 2007, ApJ, 668, 1042

Kirk, H., Pineda, J. E., Johnstone, D., \& Goodman, A. 2010, ApJ, 723, 457

Koch, E., Rosolowsky, E., \& Leroy, A. K. 2018, Res. Notes. Am. Astron. Soc., 2, 220

Ladjelate, B., André, P., \& Könyves, V. 2020, A\&A, 638, A74

Leroy, A. K., Hughes, A., Schruba, A., et al. 2016, ApJ, 831, 16

Loren, R. B., Wootten, A., \& Wilking, B. A. 1990, ApJ, 365, 269

Mangum, J. G., Emerson, D. T., \& Greisen, E. W. 2007, A\&A, 474, 679

Markwardt, C. B. 2009, , D. A. Bohlender, D. Durand, \& P. Dowler, ASP Conf. Ser., 411, 251

Motte, F., Andre, P., \& Neri, R. 1998, A\&A, 336, 150

Myers, P. C. 1983, ApJ, 270, 105

Myers, P. C., \& Benson, P. J. 1983, ApJ, 266, 309

Ortiz-León, G. N., Loinard, L., Dzib, S. A., et al. 2018, ApJ, 869, L33

Pineda, J. E., Goodman, A. A., Arce, H. G., et al. 2010, ApJ, 712, L116

Rosolowsky, E. W., Pineda, J. E., Foster, J. B., et al. 2008, ApJS, 175, 509

Walsh, A. J., Myers, P. C., Di Francesco, J., et al. 2007, ApJ, 655, 958

Williams, J. P., \& Myers, P. C. 2000, ApJ, 537, 891 


\section{Appendix A: Calculation of non-thermal velocity dispersion, $\sigma_{\mathrm{NT}}$}

The non-thermal component of the velocity dispersion is calculated by removing the thermal dispersion for the observed molecule $\left(\sigma_{\mathrm{T}}\right)$ from the total observed velocity dispersion $\left(\sigma_{\mathrm{v}}\right)$ :

$\sigma_{\mathrm{NT}}^{2}=\sigma_{\mathrm{v}}^{2}-\sigma_{T, \mathrm{NH}_{3}}^{2}-{\sigma_{\text {chan }}}^{2}$,

where $\sigma_{\text {chan }}$ is the contribution due to the width of the channel and the thermal component of the dispersion observed in $\mathrm{NH}_{3}$ is

$\sigma_{T, \mathrm{NH}_{3}}=\sqrt{\frac{k_{\mathrm{B}} T_{k}}{\mu_{\mathrm{NH}_{3}}}}$,

with $\mu_{\mathrm{NH}_{3}}=17 \mathrm{amu}$, being the mass of the ammonia molecule. The typical values of $T_{\mathrm{K}}$ and $\sigma_{\mathrm{v}}$ in this study are $14 \mathrm{~K}$ and $0.2 \mathrm{~km} \mathrm{~s}^{-1}$, respectively (see Sect. 4.1). At $T_{\mathrm{K}}=14 \mathrm{~K}$, the thermal component, $\sigma_{T, \mathrm{NH}_{3}}$ is $0.082 \mathrm{~km} \mathrm{~s}^{-1}$.

When a Hann-like kernel is used in the spectrometer, then the correction factor for the measured velocity dispersion is given by (see Leroy et al. 2016; Koch et al. 2018)

$\sigma_{\text {chan }}=\frac{\Delta}{\sqrt{2 \pi}}\left(1.0+1.18 k+10.4 k^{2}\right)$

where $\Delta$ is the spectral resolution and $k$ is dependent on the Hann-like function applied. In the case of the VEGAS spectrometer used in the GAS observations, $k$ is 0.11 , which corresponds to a correction term of

$\sigma_{\text {chan }}=\frac{\Delta}{1.994}=0.036 \mathrm{~km} \mathrm{~s}^{-1}$.

After applying this small correction, we obtain a typical non-thermal component of the velocity dispersion, $\sigma_{\mathrm{NT}}=$ $0.182 \mathrm{~km} \mathrm{~s}^{-1}$.

\section{Appendix B: Coherent cores}

As mentioned in Sect. 3.2, we identify 12 regions in L1688 as coherent cores. These include:

- five cores, Oph-A, Oph-C, Oph-D, Oph-E and Oph-F, which were identified in continuum emission (Motte et al. 1998),

- one $\mathrm{DCO}^{+}$core, Oph-B3 (Loren et al. 1990; Friesen et al. 2009)

- two coherent "droplets", L1688-d10 and L1688-d12, (Chen et al. 2019), and

- one prestellar core, Oph-H-MM1 (Johnstone et al. 2004). Apart from these previously identified cores, we also identified three more subsonic regions : L1688-SR1 (south of Oph-B1 in Motte et al. 1998), L1688-SR2 (three islands east of Oph-A), and L1688-SR3 (near the western edge of the map). Ladjelate et al. (2020) identifies a prestellar core at the position of SR1 and an unbound starless core at the position of SR3. SR1 and $\mathrm{SR} 3$ are associated with a local peak in $\mathrm{NH}_{3}$ integrated intensity. SR2 does not contain any bound core in the Herschel data, nor does it show a local $\mathrm{NH}_{3}$ peak.

Oph-B3, Oph-D and H-MM1 are also identified as L1688c4, L1688-d11 and L1688-d9, respectively, in Chen et al. (2019). The two structures that we identify as Oph-A are likely associated with Oph A-N2 and Oph A-N6, as identified in $\mathrm{N}_{2} \mathrm{H}^{+}$by Di Francesco et al. (2004). The continuum cores Oph-B1 and Oph-B2 from Motte et al. (1998) are not subsonic (Friesen et al. 2009) and, therefore, they are not considered as "coherent cores" by our definition.

\section{Appendix C: Checking effect of smoothing the data on the final results}

To check if there is any effect due to smoothing the data (see Sect. 2), we performed the same averaging on the original GAS data, using the velocity map published in DR1 for aligning the spectra. Since the DR1 velocity map was not as extensive, this test could not be performed for all the cores, as we need the velocity information to align the spectra. In the cores where this check was possible, the results from the new fits were not significantly different $\left(\approx 1.4 \%\right.$ change in $T_{\mathrm{K}}$ and $\approx 4.7 \%$ change in $\left.\sigma_{\mathrm{V}}\right)$. In comparison, the difference in the estimates from the narrow component and from single-component fit results were $10 \%$ in $T_{\mathrm{K}}$ and $28 \%$ in $\sigma_{\mathrm{v}}$ (Sect. 4.4).

\section{Appendix D: Fitting the average spectra in the cores}

To fit the average spectra in cores, we followed the same process as described in Sect. 3.1. We used the module specfit in pyspeckit to fit single spectra. Since we were fitting the spectra towards the cores, we used, as our initial guess, slightly lower values of $12 \mathrm{~K}$ and $4 \mathrm{~K}$ for $T_{\mathrm{K}}$ and $T_{\mathrm{ex}}$, respectively. As the spectra are aligned at $v=0$, we used $v_{\mathrm{LSR}}=0.0 \mathrm{~km} \mathrm{~s}^{-1}$ as our initial guess. For $\sigma_{\mathrm{v}}$, we used the mean value inside all cores, which is $\sigma_{\mathrm{v}} \approx 0.19 \mathrm{~km} \mathrm{~s}^{-1}$, from the extended velocity dispersion map obtained from the fit process described in Sect. 3.1.

While fitting two components to the spectra, we added a twocomponent cold_ammonia model as a new model in the fitter. We then used this model to fit the spectra. As the residuals from single-component fits suggest the presence of a narrow and a broad component, we used two values, one from each side of the guess used in the single-component fit $\left(0.19 \mathrm{~km} \mathrm{~s}^{-1}\right)$ as our initial $\sigma_{\mathrm{v}}$ guesses for the two components. After checking the fit with varying initial guesses, we found that small differences in $T_{\mathrm{K}}$ and $v_{\mathrm{LSR}}$ in the initial guesses also aid in arriving at a stable fit. Therefore, we used the following initial guesses in the twocomponent fits:

$-\left(T_{\mathrm{K}}\right)_{1}=11 \mathrm{~K}$

$-\left(T_{\mathrm{ex}}\right)_{1}=4 \mathrm{~K}$

$-\left(\sigma_{\mathrm{v}}\right)_{1}=0.15 \mathrm{~km} \mathrm{~s}^{-1}$

$-\left(v_{\mathrm{LSR}}\right)_{1}=0.0 \mathrm{~km} \mathrm{~s}^{-1}$

$-\left(T_{\mathrm{K}}\right)_{2}=16 \mathrm{~K}$

$-\left(T_{\mathrm{ex}}\right)_{2}=4 \mathrm{~K}$

$-\left(\sigma_{\mathrm{v}}\right)_{2}=0.3 \mathrm{~km} \mathrm{~s}^{-1}$

$-\left(v_{\mathrm{LSR}}\right)_{2}=0.25 \mathrm{~km} \mathrm{~s}^{-1}$.

\section{Appendix E: Model selection using AIC estimator}

We fitted the averaged spectra considering models with a different number of components in ammonia, with each component being modelled as a cold_ammonia spectrum in pyspeckit. The number of parameters used in the models is five per component. To select the best model to fit a spectra, we checked the Akaike Information Criterion (AIC), which determines if the quality of the model significantly improves considering the increase in the number of parameters used. Assuming that each channel in the spectra has a constant Gaussian error, which is equal to the noise in the spectra, AIC is related to the $\chi^{2}$ of the data as:

$\mathrm{AIC}=2 k+\chi^{2}+C$,

where $k$ is the number of parameters used to model the data and $C$ is a constant, which depends on the noise in the spectra, and is 
given by :

$C=-2 N \times \ln \left(\frac{1}{\sqrt{2 \pi \sigma^{2}}}\right)$,

where $N$ is the number of channels in the spectra.

The model with the lowest AIC value is considered to be the best model. In our case, the channels are not completely independent and, therefore the actual number of independent channels is different. This effect, however, is systematic and it does not change for models with different number of components. The change in the AIC value from one model to another is the important term, and the actual AIC values are less significant.

Using Eq. (E.1) we can rewrite the change in AIC value from a single-component fit to a two-component fit, $\Delta_{\mathrm{AIC}}$, as

$\Delta_{\mathrm{AIC}}=2 \Delta k+\Delta \chi^{2}$,

where $\Delta k$ is the change in number of parameters and $\Delta \chi^{2}$ is the change in $\chi^{2}$, from a single-component fit to a two-component fit. Each model component uses five independent parameters and, therefore $\Delta k=-5$. Also, $\Delta \chi^{2}$ can be divided into two groups: channels with and without emission, or

$\Delta \chi^{2}=\left(\Delta \chi^{2}\right)_{\text {with emission }}+\left(\Delta \chi^{2}\right)_{\text {without emission }}$.

Outside the emission region the models are identical and, therefore,

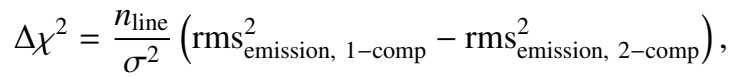

where, $n_{\text {line }}$ is the number of channels with emission, $\sigma$ is the noise in the spectra, and $\mathrm{rms}_{\text {emission }}$ is the residual root mean square (rms) of the model in the emission region. The comparison of the $\sigma_{\text {emission }}$ and the noise, $\sigma$, is taken as a measure of determining the goodness of the fit in line-fitting software, such as CLASS in GILDAS ${ }^{6}$. Then, in our comparison of single- and two-component fits, we have

$\Delta_{\mathrm{AIC}}=-10+\frac{n_{\text {line }}}{\sigma^{2}}\left(\mathrm{rms}_{\text {emission, 1-comp }}^{2}-\mathrm{rms}_{\text {emission, 2-comp }}^{2}\right)$.

We note that $n_{\text {line }}$ depends on the width of the spectra and, therefore, it is different for each coherent core. From Eq. (E.6), we see that for a low variation in the AIC, the improvement in the residuals of the fit is also small. We find that for the two coherent cores with low $\Delta_{\mathrm{AIC}}(<30), \mathrm{d} 12$ and SR3, the improvement in the $\mathrm{rms}_{\text {emission }}$ is less than $10 \%$ of the noise in the respective spectra. For the ambient cloud spectra, with $\Delta_{\mathrm{AIC}}=94, \mathrm{rms}_{\text {emission }}$ improves by $10 \%$ of the noise level, from a single-component to a two-component fit. For every other region, we see an improvement in $\mathrm{rms}_{\text {emission }}$ by $>15 \%$.

Owing to our limited number of data points, it is not possible to definitively determine what change in AIC represents a significant improvement in the fit upon the addition of the second component. From the corresponding improvement in $\mathrm{rms}_{\text {emission, we }}$ note that in our current work, only the regions with $\Delta_{\text {AIC }}>150$ show a significant improvement in the fit.

\section{Appendix F: Average spectra of the whole cloud}

Figure F.1 shows the average $(1,1)$ and $(2,2)$ spectra in the whole L1688, which was mapped by the Green Bank Ammonia Survey (GAS). These spectra were used to determine the range of centroid velocity, for which a fit is attempted with pyspeckit.
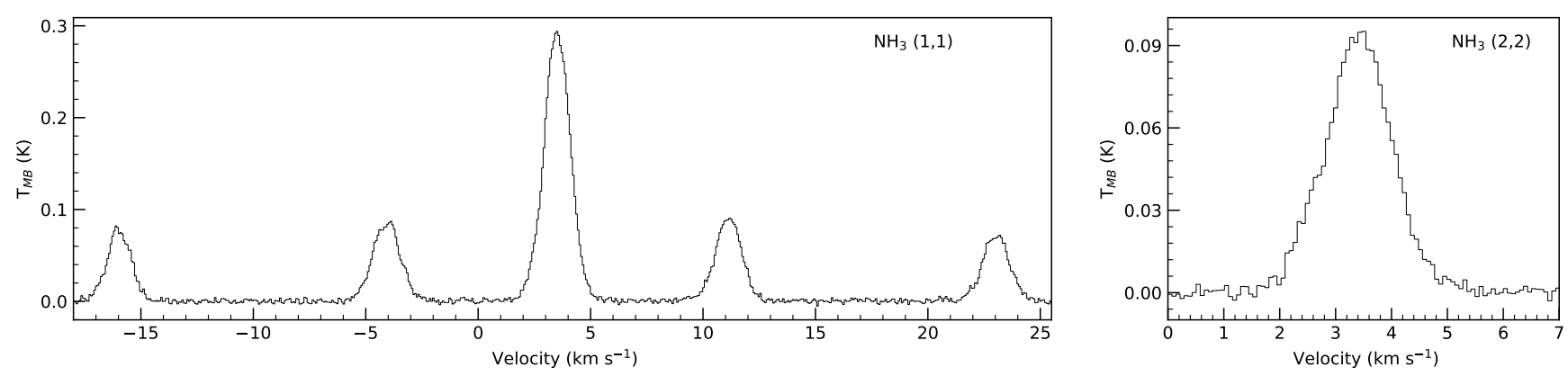

Fig. F.1. Average $\mathrm{NH}_{3}(1,1)$ and $(2,2)$ spectra in L1688. 


\section{Appendix G: Averaged spectra towards cores}

Figures G.1-G.11 show the average spectra in all of the other coherent cores studied here, with single- and two-component fits, similar to Oph-F in Fig. 2. The kinetic temperature, velocity dispersion, centroid velocity and para-ammonia column densities determined from these single- and two-component fits are presented in Table H.1. The rms noise in the spectra of each core is also shown in the same table.
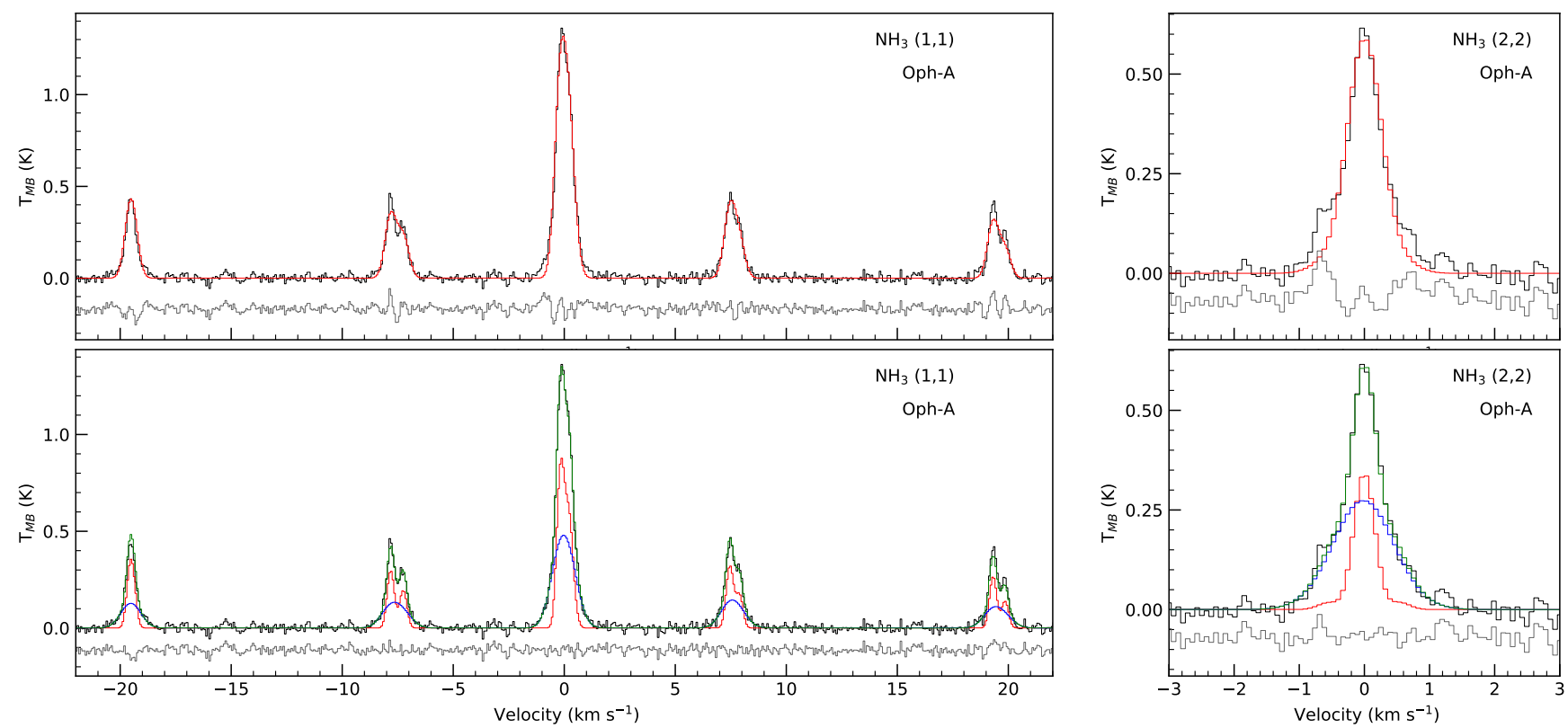

Fig. G.1. Top panels: average $\mathrm{NH}_{3}(1,1)$ and $(2,2)$ spectra of Oph-A core, with a single-component fit. Bottom panels: same spectra, with twocomponent fit (green). The narrow (red) and broad (blue) components are also shown separately.
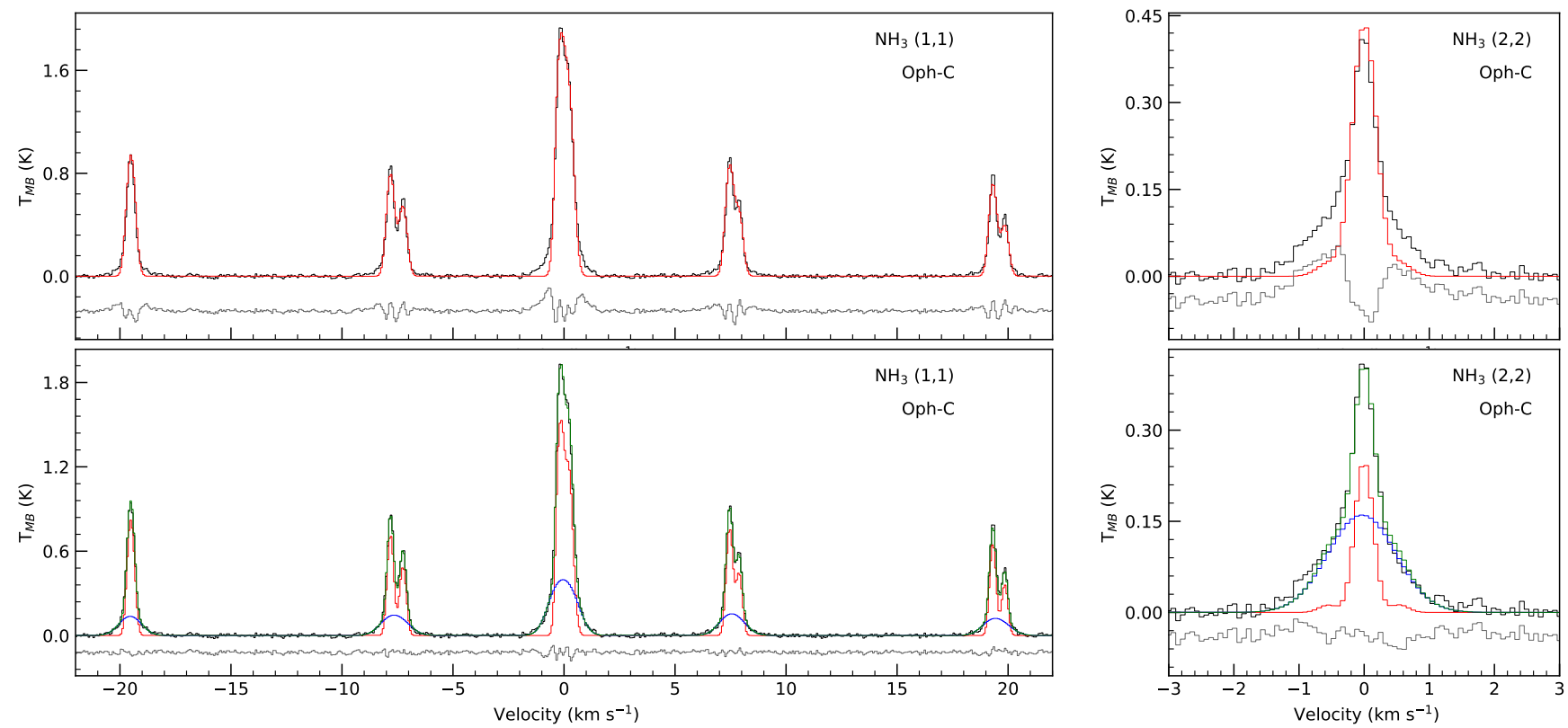

Fig. G.2. Same as Fig. G.1, but for Oph-C. 
A\&A 640, L6 (2020)
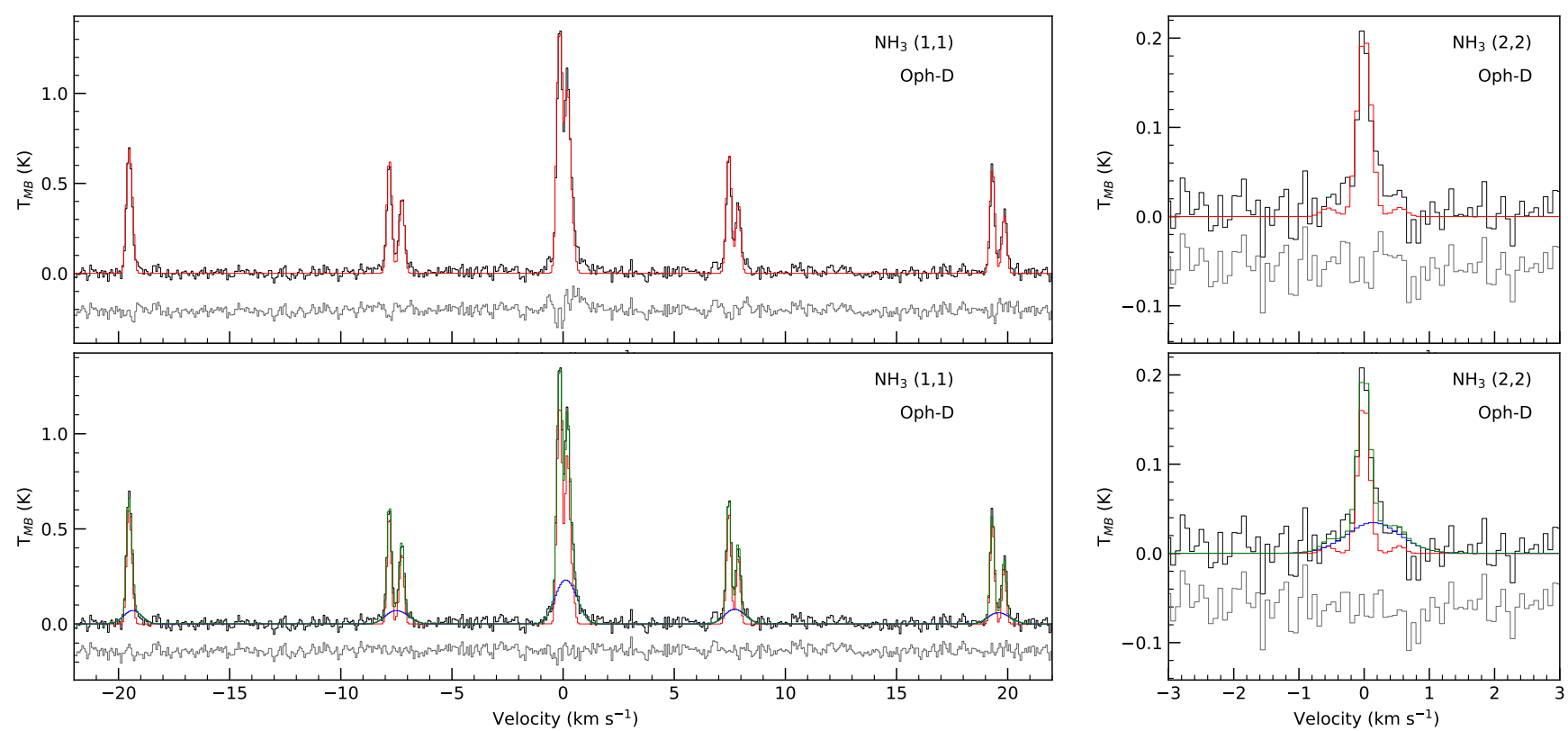

Fig. G.3. Same as Fig. G.1, but for Oph-D.
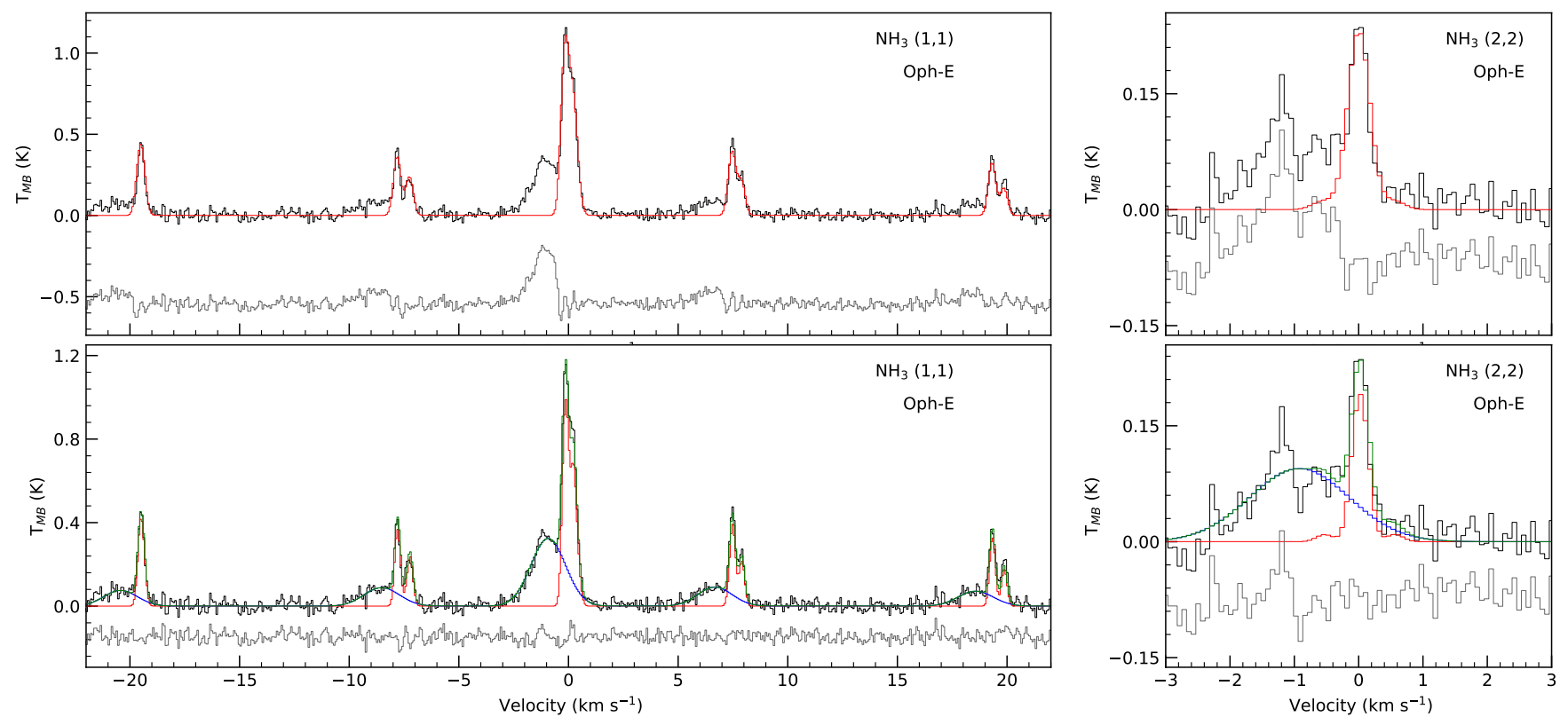

Fig. G.4. Same as Fig. G.1, but for Oph-E. 
S. Choudhury et al.: Ubiquitous $\mathrm{NH}_{3}$ supersonic component in $\mathrm{L} 1688$ coherent cores
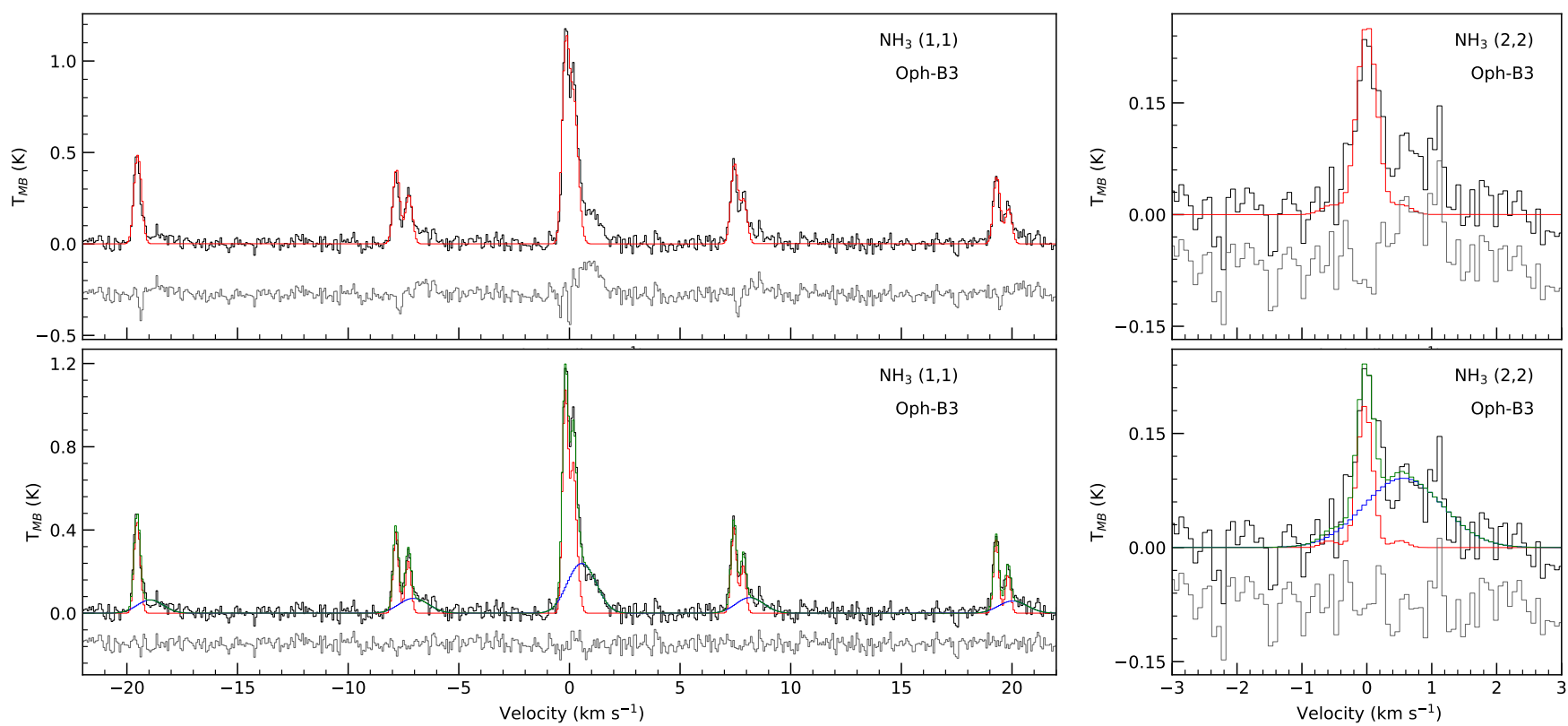

Fig. G.5. Same as Fig. G.1, but for Oph-B3.
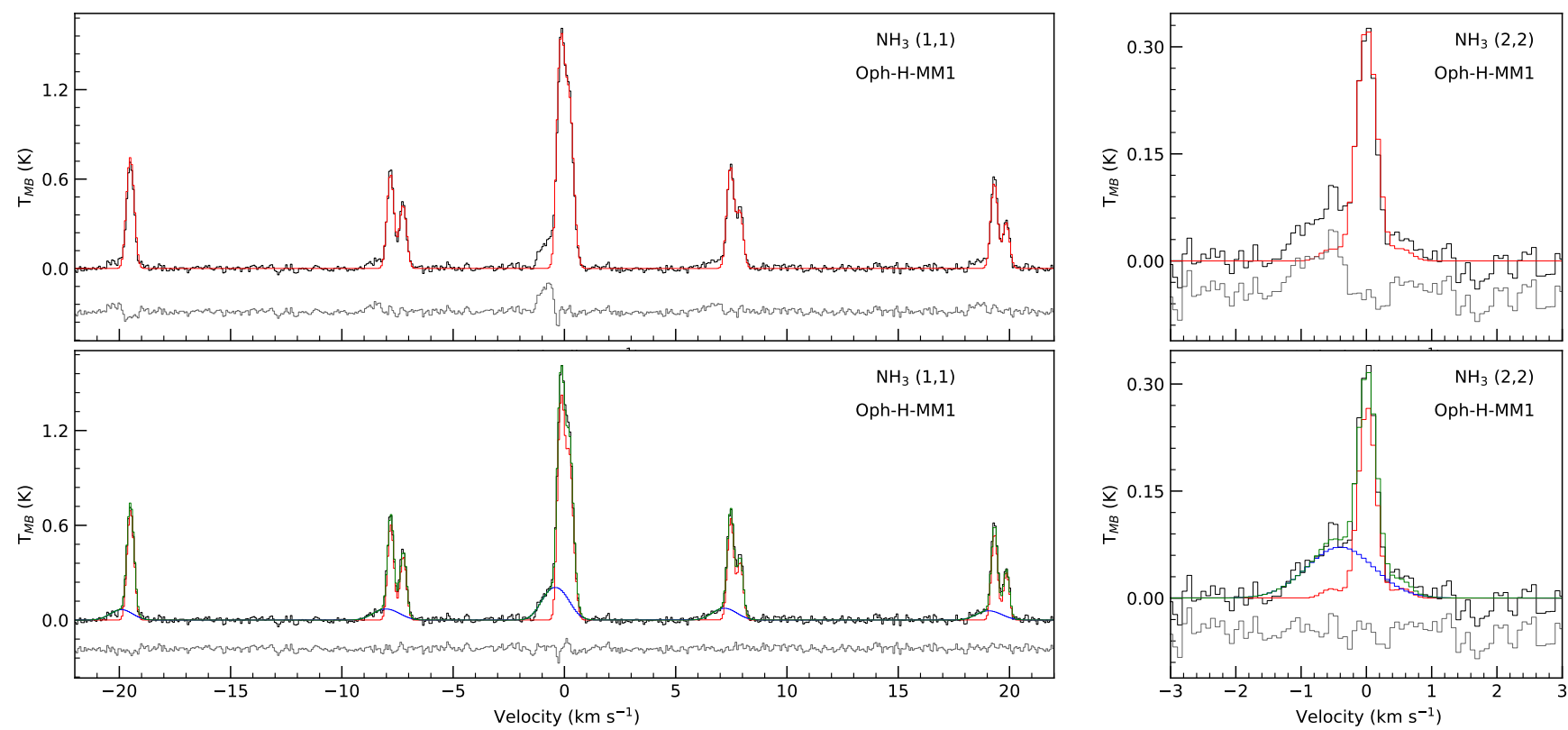

Fig. G.6. Same as Fig. G.1, but for Oph-H-MM1. 
A\&A 640, L6 (2020)
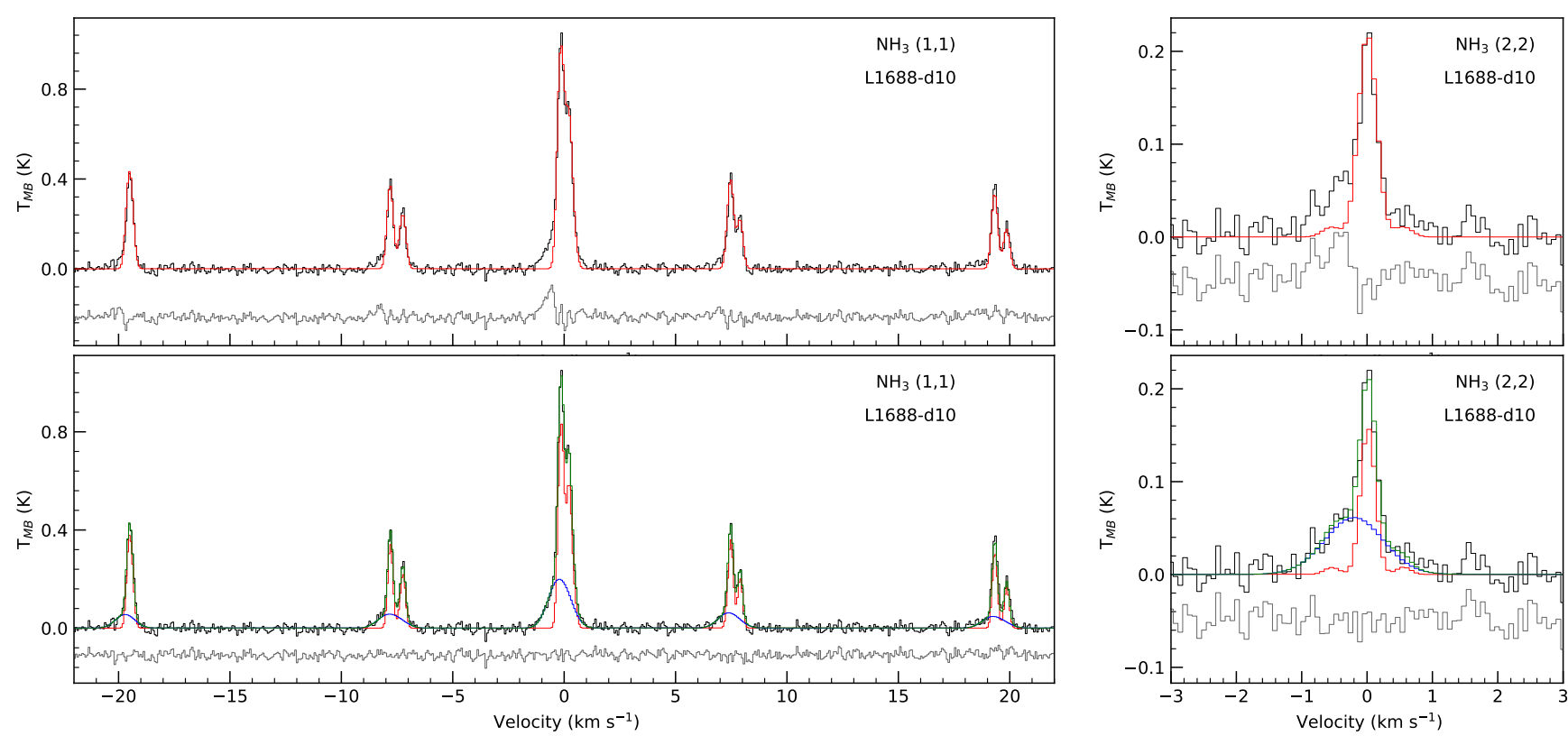

Fig. G.7. Same as Fig. G.1, but for L1688-d10.
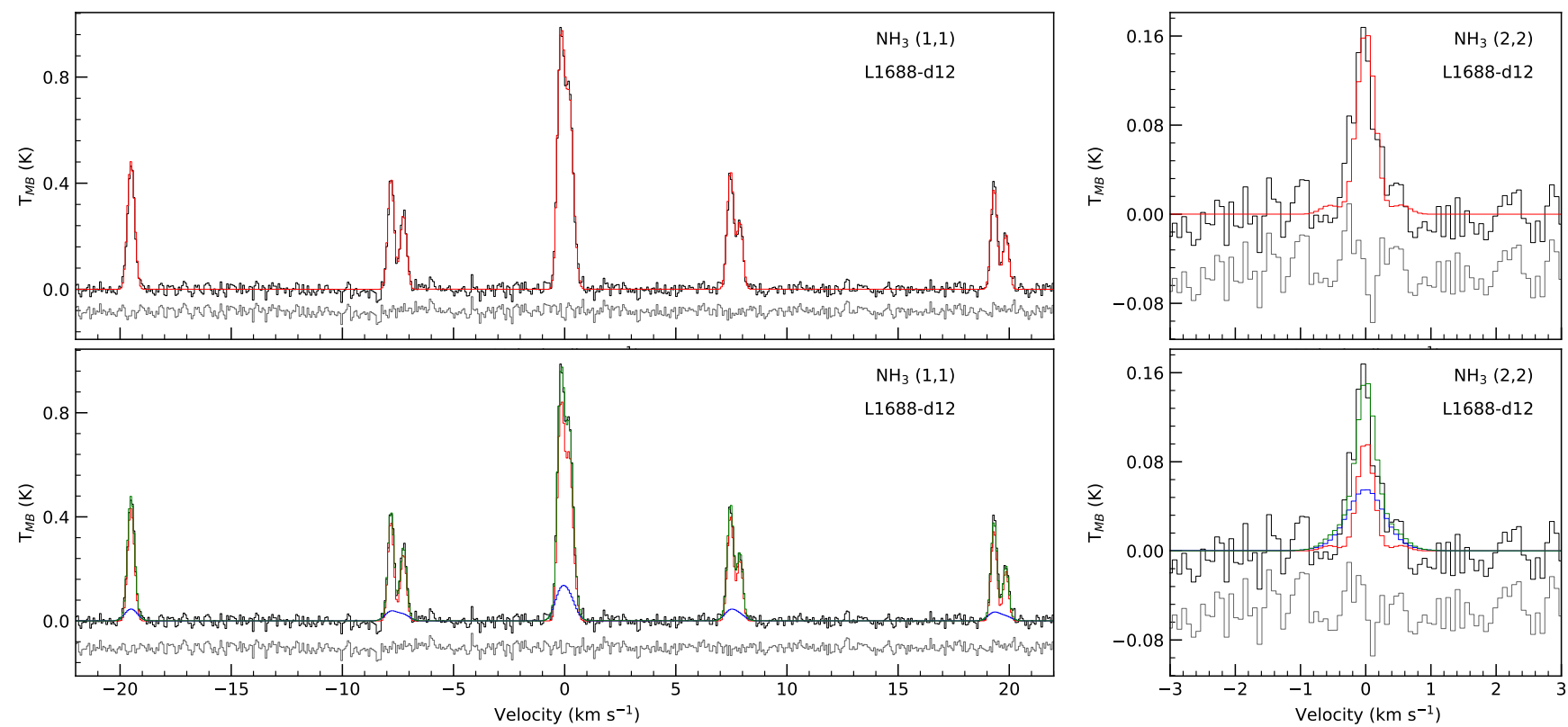

Fig. G.8. Same as Fig. G.1, but for L1688-d12. 
S. Choudhury et al.: Ubiquitous $\mathrm{NH}_{3}$ supersonic component in $\mathrm{L} 1688$ coherent cores
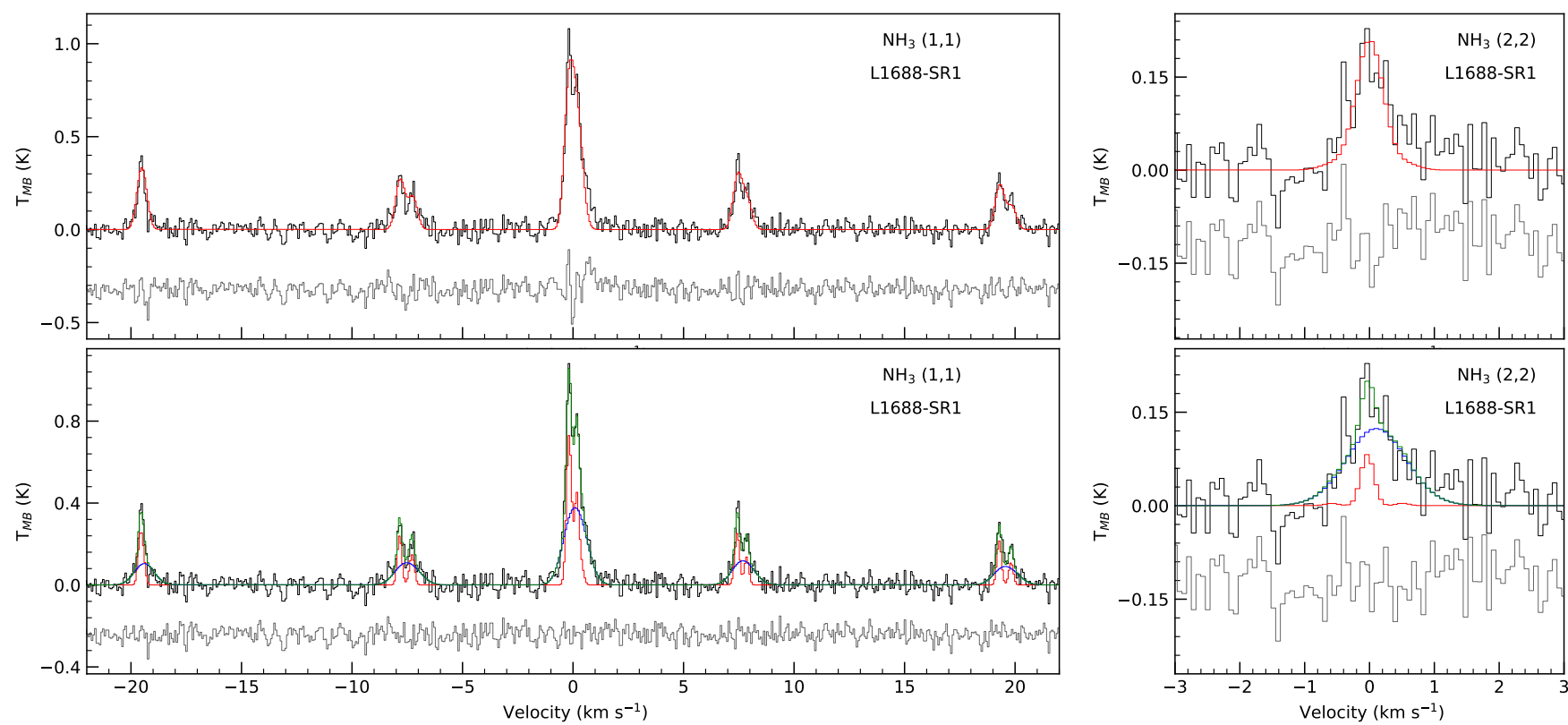

Fig. G.9. Same as Fig. G.1, but for L1688-SR1.
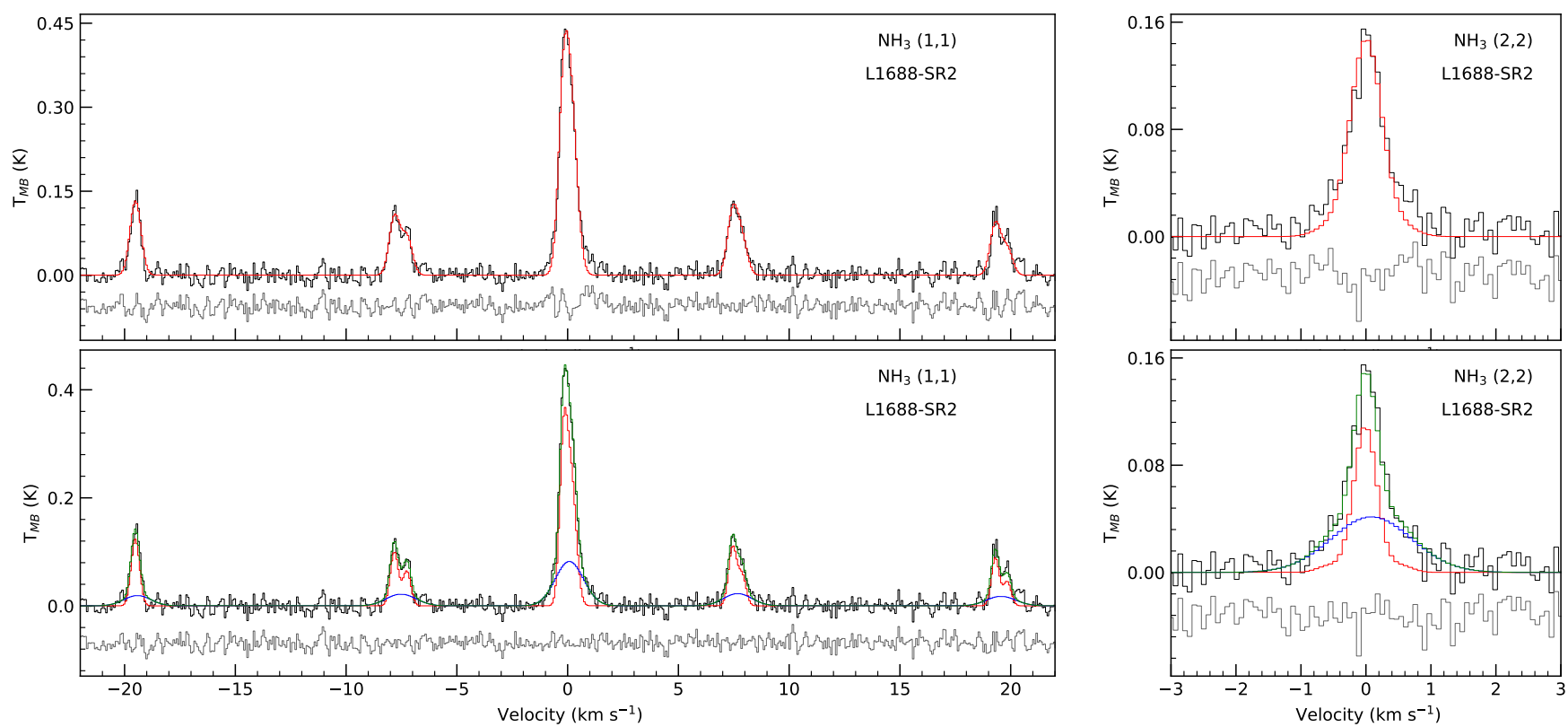

Fig. G.10. Same as Fig. G.1, but for L1688-SR2. 
A\&A 640, L6 (2020)
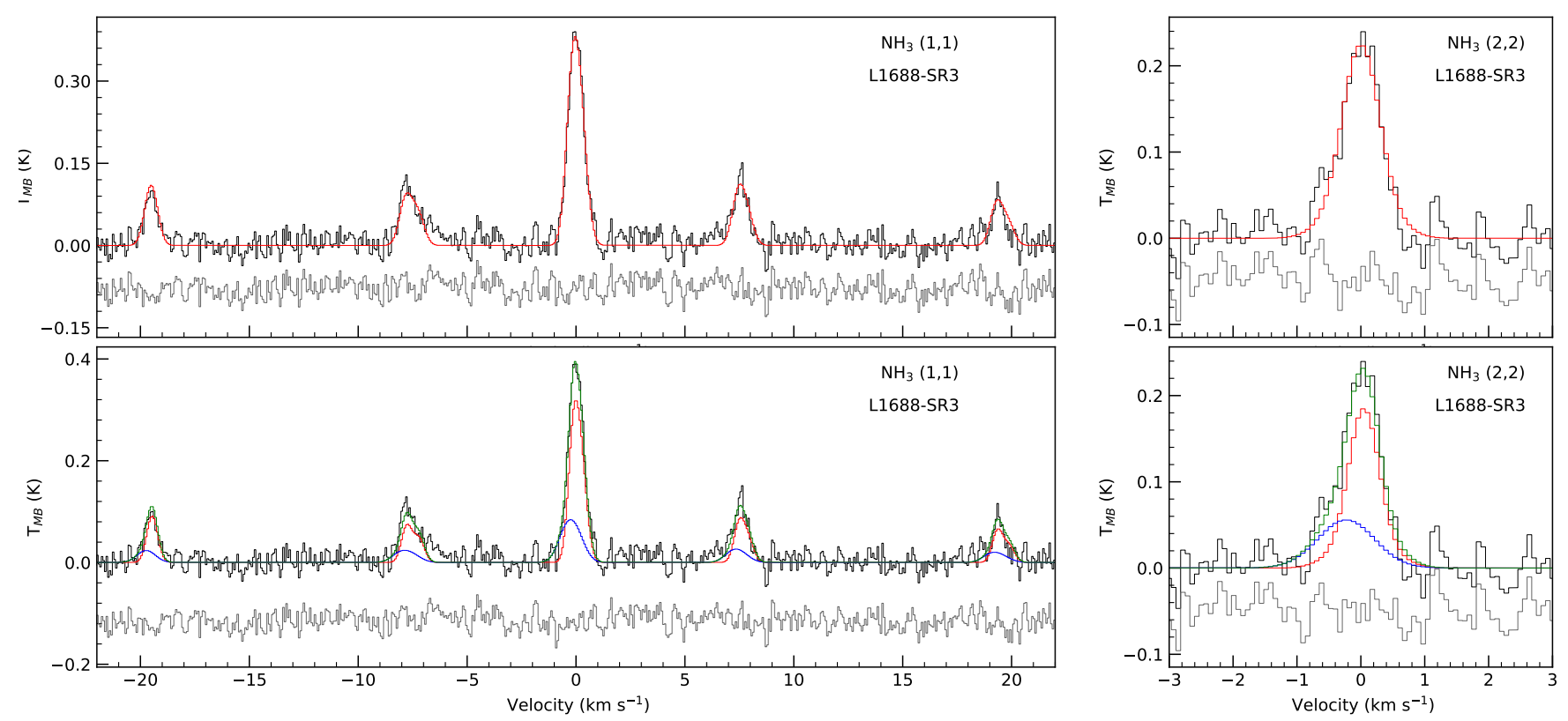

Fig. G.11. Same as Fig. G.1, but for L1688-SR3. 


\section{Appendix H: Table}

Table H.1. Best fit parameters for single- and two-component fits.

\begin{tabular}{|c|c|c|c|c|c|c|c|c|c|c|}
\hline Core & Component ${ }^{(a)}$ & $\begin{array}{l}T_{\mathrm{K}} \\
(\mathrm{K}) \\
\end{array}$ & $\begin{array}{c}\sigma_{\mathrm{v}}{ }^{(b)} \\
\left(\mathrm{km} \mathrm{s}^{-1}\right)\end{array}$ & $\begin{array}{c}v_{\text {rel }}(c) \\
\left(\mathrm{km} \mathrm{s}^{-1}\right)\end{array}$ & $\begin{array}{c}\log \left(N\left(p-\mathrm{NH}_{3}\right)\right) \\
\left(\mathrm{cm}^{-2}\right)\end{array}$ & $\mathcal{M}_{s}$ & $\begin{array}{c}\text { Noise }^{(d)} \\
(\mathrm{mK})\end{array}$ & $\Delta_{\mathrm{AIC}}{ }^{(e)}$ & $\begin{array}{c}T_{K, \mathrm{GAS}} \\
(\mathrm{K})\end{array}$ & $\begin{array}{r}\sigma_{\mathrm{v}, \mathrm{GAS}} \\
\left(\mathrm{km} \mathrm{s}^{-1}\right)\end{array}$ \\
\hline \multirow{3}{*}{ Oph-A } & Single & 19.1(2) & $0.253(3)$ & $0.0^{(f)}$ & $13.86(2)$ & 0.9 & \multirow{3}{*}{17} & \multirow{3}{*}{761} & $19(1)$ & $0.23(1)$ \\
\hline & Narrow & $15.8(4)$ & $0.156(4)$ & $-0.001(2)$ & $13.74(4)$ & 0.6 & & & & \\
\hline & Broad & $24.9(8)$ & $0.44(1)$ & $-0.014(6)$ & $-(g)$ & 1.5 & & & & \\
\hline \multirow{3}{*}{ Oph-C } & Single & 11.72(9) & $0.177(1)$ & $0.0^{(f)}$ & $14.168(5)$ & 0.8 & \multirow{3}{*}{9} & \multirow{3}{*}{6724} & $12.1(5)$ & $0.174(8)$ \\
\hline & Narrow & $10.13(9)$ & $0.1376(8)$ & $-0.0015(4)$ & $14.088(6)$ & 0.6 & & & & \\
\hline & Broad & 17.7(3) & $0.477(7)$ & $-0.033(5)$ & $14.13(3)$ & 1.9 & & & & \\
\hline \multirow{3}{*}{ Oph-D } & Single & $9.9(2)$ & $0.104(1)$ & $0.0005(5)$ & $13.94(1)$ & 0.4 & \multirow{3}{*}{22,21} & \multirow{3}{*}{383} & $10.2(4)$ & $0.116(7)$ \\
\hline & Narrow & $9.7(3)$ & $0.088(1)$ & $-0.006(1)$ & $13.89(2)$ & 0.3 & & & & \\
\hline & Broad & $12.0(1)$ & $0.39(2)$ & $0.14(2)$ & $13.8(1)$ & 1.8 & & & & \\
\hline \multirow{3}{*}{ Oph-E } & Single & $12.5(4)$ & $0.168(4)$ & $0.000(2)$ & $13.77(4)$ & 0.7 & \multirow{3}{*}{24} & \multirow{3}{*}{3698} & $12.3(5)$ & $0.171(8)$ \\
\hline & Narrow & 11.7(3) & $0.126(2)$ & $0.023(2)$ & 13.73(3) & 0.5 & & & & \\
\hline & Broad & $17.9(6)$ & $0.76(2)$ & $-0.92(3)$ & $-(g)$ & 3.0 & & & & \\
\hline \multirow{3}{*}{ Oph-F } & Single & $13.9(1)$ & $0.174(2)$ & $0.0003(5)$ & 13.91(1) & 0.7 & \multirow{3}{*}{9,11} & \multirow{3}{*}{5244} & $14.9(8)$ & $0.19(1)$ \\
\hline & Narrow & $12.6(1)$ & $0.128(1)$ & $0.006(1)$ & 13.81(1) & 0.5 & & & & \\
\hline & Broad & 19.1(4) & $0.55(1)$ & $-0.144(9)$ & $13.87(7)$ & 2.1 & & & & \\
\hline \multirow{3}{*}{ Oph-H-MM1 } & Single & $11.6(1)$ & $0.15(1)$ & $0.0008(5)$ & $14.0(9)$ & 0.6 & \multirow{3}{*}{16} & \multirow{3}{*}{1489} & $12.4(4)$ & $0.16(1)$ \\
\hline & Narrow & $11.0(1)$ & $0.133(1)$ & $0.012(1)$ & $13.971(9)$ & 0.6 & & & & \\
\hline & Broad & $16.9(7)$ & $0.47(2)$ & $-0.4(3)$ & $13.99(8)$ & 1.9 & & & & \\
\hline \multirow{3}{*}{ L1688-d10 } & Single & $12.1(2)$ & $0.137(2)$ & $0.005(1)$ & $13.79(2)$ & 0.6 & \multirow{3}{*}{14} & \multirow{3}{*}{826} & $12.2(2)$ & $0.160(8)$ \\
\hline & Narrow & 11.2(2) & $0.111(2)$ & $0.015(2)$ & $13.76(2)$ & 0.4 & & & & \\
\hline & Broad & 17.1(8) & $0.42(2)$ & $-0.2(2)$ & $13.6(2)$ & 1.7 & & & & \\
\hline \multirow{3}{*}{ L1688-d12 } & Single & $10.6(2)$ & $0.135(1)$ & $-0.0004(4)$ & $13.92(1)$ & 0.6 & \multirow{3}{*}{16} & \multirow{3}{*}{30} & $10.4(1)$ & $0.129(6)$ \\
\hline & Narrow & $9.4(6)$ & $0.123(4)$ & $-0.0009(7)$ & 13.91(3) & 0.6 & & & & \\
\hline & Broad & $18.0(3)$ & $0.26(5)$ & $0.0(1)$ & $13.6(4)$ & 1.0 & & & & \\
\hline & Single & $12.4(3)$ & $0.149(3)$ & $-0.002(2)$ & $13.81(3)$ & 0.6 & & & $12.5(5)$ & $0.14(1)$ \\
\hline Oph-B3 & Narrow & $11.4(3)$ & $0.117(2)$ & $-0.028(2)$ & $13.67(3)$ & 0.5 & 26,28 & 1083 & & \\
\hline & Broad & $19.0(1)$ & $0.58(3)$ & $0.56(4)$ & $13.8(2)$ & 2.2 & & & & \\
\hline & Single & $13.5(4)$ & $0.209(6)$ & $0.000(1)$ & $13.75(5)$ & 0.9 & & & $14.2(5)$ & $0.18(2)$ \\
\hline L1688-SR1 & Narrow & $10(1)$ & $0.102(5)$ & $-0.033(4)$ & 13.3(1) & 0.4 & 35 & 308 & & \\
\hline & Broad & $18.0(1)$ & $0.43(2)$ & $0.11(2)$ & 13.7(2) & 1.7 & & & & \\
\hline & Single & $17.2(3)$ & $0.239(4)$ & $0.000(2)$ & $13.35(8)$ & 0.9 & & & $16.70(6)$ & $0.222(3)$ \\
\hline L1688-SR2 & Narrow & $15.4(5)$ & $0.178(6)$ & $-0.009(4)$ & 13.2(1) & 0.7 & 11 & 164 & & \\
\hline & Broad & $24.0(2)$ & $0.6(5)$ & $0.08(3)$ & $13.0(4)$ & 2.0 & & & & \\
\hline & Single & $24.6(6)$ & $0.295(8)$ & $0.0^{(f)}$ & $-(g)$ & 0.9 & & & $-{ }^{(h)}$ & $-{ }^{(h)}$ \\
\hline L1688-SR3 & Narrow & 24(1) & $0.24(2)$ & $0.05(2)$ & $-(g)$ & 0.8 & 18 & 12 & & \\
\hline & Broad & $27.0(5)$ & $0.41(7)$ & $-0.2(2)$ & $-(g)$ & 1.3 & & & & \\
\hline & Single & $16.9(2)$ & $0.451(4)$ & $0.0002(0)$ & $13.79(2)$ & 1.8 & & & $-{ }^{(h)}$ & $-{ }^{(h)}$ \\
\hline Ambient cloud $^{(i)}$ & Broad-1 & $16.9(2)$ & $0.35(2)$ & $-0.16(4)$ & $13.74(3)$ & 1.4 & 9 & 94 & & \\
\hline & Broad-2 & $17.0(5)$ & $0.35(3)$ & $0.45(7)$ & $13.2(3)$ & 1.4 & & & & \\
\hline
\end{tabular}

Notes. Kinetic temperatures, velocity dispersions and $p-\mathrm{NH}_{3}$ column densities, derived from single-component fits, and two-component fits, in the coherent cores and the ambient cloud ${ }^{(i)}$. The values in parentheses represent the fit-determined error in the final decimal place of the corresponding parameter. These uncertainties do not include the calibration uncertainty, which is $\sim 10 \%$. The noise level achieved in the average spectra are also shown. The final two columns show the average kinetic temperature and velocity dispersions inside the cores, from GAS DR1 (Friesen et al. 2017) results (single-component fit). ${ }^{(a)}$ single-component fit, or the individual components of the two-component fit. ${ }^{(b)}$ The channel response, $\sigma_{\text {chan }}($ see Sect. 3.2), is not removed from the $\sigma_{\mathrm{v}}$ value reported here (the contribution from $\sigma_{\text {chan }}$ is very small, changes only in the third decimal place in $\left.\sigma_{\mathrm{v}}\right) .{ }^{(c)}$ Velocity from the fit. Since we align the spectrum in the core by the velocity at each pixel (determined from single-component fit), the velocities reported in this Table are relative to the mean velocity in the corresponding core. ${ }^{(d)}$ Noise level estimated for both $\mathrm{NH}_{3}(1,1)$ and $(2,2)$. In cases where two values are reported, the noise in $\mathrm{NH}_{3}(1,1)$ and $(2,2)$ are not the same, and the values correspond to the noise in $(1,1)$ and $(2,2)$, respectively. ${ }^{(e)}$ Change in AIC, from 1-component fit to 2-component fit $\left(\Delta_{\mathrm{AIC}}=\mathrm{AIC}_{1 \text {-comp. }}-\mathrm{AIC}_{2 \text {-comp. }}\right) .{ }^{(f)}$ Value and error smaller than $10^{-4} \mathrm{~km} \mathrm{~s}^{-1} .{ }^{(g)}$ Excitation temperature could not be well-constrained from the fit (fit determined error $>30 \%$ ), and therefore, the column density estimate is not very reliable. ${ }^{(h)}$ Velocity dispersion and temperature maps from GAS DR1 did not include any pixel inside SR3, or ambient cloud box. ${ }^{(i)}$ Represents the average spectra in the "ambient cloud box" shown in Fig. 1. 